Full-length article

Manufacturing

\title{
A comprehensive report of long-term stability data for a range ATMPs: A need to develop guidelines for safe and harmonized stability studies
}

\author{
Chiara Capelli ${ }^{1,8}$, Simona Frigerio $^{2}$, Daniela Lisini ${ }^{2}$, Sara $\mathrm{Nava}^{2}$, Giuseppe Gaipa ${ }^{3}$, \\ Daniela Belotti ${ }^{3}$, Benedetta Cabiati ${ }^{3}$, Silvia Budelli ${ }^{4}$, Lorenza Lazzari ${ }^{4}$, Jessica Bagnarino ${ }^{5,6}$, \\ Matteo Tanzi ${ }^{5,6}$, Patrizia Comoli ${ }^{5,6}$, Norberto Perico ${ }^{7}$, Martino Introna ${ }^{1, *}$, Josée Golay ${ }^{1,8}$ \\ ${ }^{1}$ Center of Cellular Therapy “G. Lanzani”, ASST Papa Giovanni XXIII, Bergamo, Italy \\ ${ }^{2}$ Cell Therapy Production Unit, Fondazione IRCCS Istituto Neurologico Carlo Besta, Milan, Italy \\ ${ }^{3}$ Laboratory of Cell and Gene Therapy Stefano Verri, ASST Monza Ospedale San Gerardo, Monza, Italy \\ ${ }^{4}$ Laboratory of Regenerative Medicine - Cell Factory, Department of Transfusion Medicine and Hematology, Fondazione IRCCS Ca' Granda Ospedale Maggiore \\ Policlinico, Milano, Italy \\ ${ }^{5}$ UOSD Cell Factory, Fondazione IRCCS Policlinico San Matteo, Pavia, Italy \\ ${ }^{6}$ Pediatric Hematology/Oncology, Fondazione IRCCS Policlinico San Matteo, Pavia, Italy \\ ${ }^{7}$ Aldo \& Cele Daccò Clinical Research Center for Rare Diseases, Istituto Ricerche Farmacologiche Mario Negri IRCCS, Bergamo, Italy \\ ${ }^{8}$ Fondazione per la Ricerca Ospedale di Bergamo, Bergamo, Italy
}

\section{A R T I C L E I N F O}

\section{Article History:}

Received 21 June 2021

Accepted 6 December 2021

\section{Keywords:}

advanced therapy medicinal products

Good Manufacturing Practices

potency assays

quality controls

shelf life

stability study

\begin{abstract}
A B S T R A C T
Background aims: Advanced therapy medicinal products (ATMPs) are novel drugs based on genes, cells or tissues developed to treat many different diseases. Stability studies of each new ATMP need to be performed to define its shelf life and guarantee efficacy and safety upon infusion, and these are presently based on guidelines originally drafted for standard pharmaceutical drugs, which have properties and are stored in conditions quite different from cell products. The aim of this report is to provide evidence-based information for stability studies on ATMPs that will facilitate the interlaboratory harmonization of practices in this area. Methods: We have collected and analyzed the results of stability studies on 19 different cell-based experimental ATMPs, produced by five authorized cell factories forming the Lombardy "Plagencell network" for use in 36 approved phase I/II clinical trials; most were cryopreserved and stored in liquid nitrogen vapors for 1 to 13 years. Results: The cell attributes collected in stability studies included cell viability, immunophenotype and potency assays, in particular immunosuppression, cytotoxicity, cytokine release and proliferation/differentiation capacity. Microbiological attributes including sterility, endotoxin levels and mycoplasma contamination were also analyzed. All drug products (DPs), cryopreserved in various excipients containing $10 \%$ DMSO and in different primary containers, were very stable long term at $<-150^{\circ} \mathrm{C}$ and did not show any tendency for diminished viability or efficacy for up to 13.5 years.

Conclusions: Our data indicate that new guidelines for stability studies, specific for ATMPs and based on risk analyses, should be drafted to harmonize practices, significantly reduce the costs of stability studies without diminishing safety. Some specific suggestions are presented in the discussion.
\end{abstract}

(c) 2022 International Society for Cell \& Gene Therapy. Published by Elsevier Inc. This is an open access article under the CC BY-NC-ND license (http://creativecommons.org/licenses/by-nc-nd/4.0/)

\section{Introduction}

Extensively manipulated cells for therapeutic use are called advanced therapy medicinal products (ATMPs) and can be genetically engineered or not (somatic) [1,2]. Most ATMPs are produced by quite

\footnotetext{
* Corresponding author: Martino Introna, Center of Cellular Therapy “G. Lanzani”, ASST Papa Giovanni XXIII.

E-mail address: mintrona@asst-pg23.it (M. Introna).
}

extensive culture methods and are generally cryopreserved at $<-150^{\circ} \mathrm{C}$ in liquid nitrogen vapors to allow time for the execution of all quality controls on the finished product before administration to patients and to allow multiple dosing [3-6]. By law, they need to be produced according to specific guidelines and regulations (e.g., the EU Good Manufacturing Practices [GMP] guidelines, published on the Eudralex website, and the U.S. Food and Drug Administration [FDA] current GMP regulations [cGMP], published on the FDA website) [7]. In the European Union, the same GMP regulations already established 
for standard drugs were initially applied to ATMPs [8,9]. In 2017, a specific GMP guideline dedicated to ATMPs was approved by the European Commission (EC) [10], since it is understood that cells and tissues are quite different from standard pharmaceutical drugs [4].

Stability studies determine a drug product (DP) "shelf life," i.e. they guarantee that the final packaged DP maintains its identity, potency and sterility according to the approved manufacturer's specification throughout its valid period, in defined storage conditions [11-13]. Present guidelines and regulations prescribe that preliminary stability data should be available before an experimental product is used in a clinical trial, a minimum shelf life should be assigned, and further stability data should be planned and built up with reallife data subsequent to initial clinical use, eventually extending shelf life $[6,14]$. Unfortunately, the current guidance documents do not offer much support to establish the best methods and modalities of testing ATMP stability. This lack of formal advice can lead to uncertainty and different practices in different cell factories and different countries. Furthermore, the many assays to control DPs often are not standardized or harmonized between laboratories.

An official network of the five academic cell factories, called "Plagencell," all of which are authorized by Italy's national authorities (Agenzia Italiana del Farmaco, AIFA, Rome, Italy), has recently been established in the Lombardy region. As a first investigation, the Plagencell network has set out to collect and analyze the stability studies performed in the last 13 years on the experimental ATMPs internally produced for approved phase I/II clinical studies. The aim was to try to pinpoint the major bottlenecks and propose specific elements useful for the future establishment of guidelines more specific for this type of DPs, based on scientific evidence and real-life data.

\section{Methods}

\section{The Plagencell network of cell factories}

The Plagencell Cell Factories are numbered in the text and figures as follows: CF1, Center of Cellular Therapy "G. Lanzani", ASST Papa Giovanni XXIII, Bergamo; CF2, Laboratory of Cellular and Gene Therapy “S. Verri”, ASST San Gerardo, Monza; CF3, Laboratorio di Medicina Rigenerativa - Cell Factory, Fondazione IRCCS Ca' Granda Ospedale Maggiore Policlinico, Milan; CF4, Cell Therapy Production Unit UPTC, IRCCS Istituto Neurologico Carlo Besta, Milan; and CF5, UOSD Cell Factory, Fondazione IRCCS Policlinico San Matteo, Pavia.

\section{ATMP manufacturing, cryopreservation and thawing}

Different types of experimental ATMPs were produced in GMP conditions following validated standard operating procedures (SOPs). These were either stored at $2^{\circ}$ to $8^{\circ} \mathrm{C}$ until use or cryopreserved in different solutions (human Plasma $\mathrm{AB}$ or saline solution supplemented with human serum albumin (HSA) containing 10\% dimethyl sulfoxide (DMSO, GMP grade; WAK-Chemie Medica, Steinbach, Germany; or Cryoserv; Mylan Institutional, Canonsburg, PA, or Alchimia, Ponte San Nicolò, Italy) or Cryostor CS10 (BioLife Solutions, Bothell, WA) [15-18]. Cells were frozen in double clinical bags (CryoMACS Freezing bag; Miltenyi Biotec, Bergisch Gladbach, Germany; or SAFE2 Cell Cryobag; Paolo Gobbi Frattini, Tovo di S. Agata, Italy; or HemoFreeze bag; Fresenius Kabi, Bad Homburg, Germany) or cryovials (Nalgene, Thermo Scientific, Waltham, MA) using controlled-rate freezing instruments and stored in vapor-phase liquid nitrogen storage tanks. The liquid nitrogen freezers were connected to an automated filling system and equipped with remote alarm systems; temperature and nitrogen levels were monitored and registered continuously.

Tubes or bags were thawed in a thermostatic bath at $37^{\circ} \mathrm{C}$ or dry thawing device; products were in some cases diluted in $\mathrm{NaCl} 0.9 \%$ supplemented with HSA to evaluate cellular viability and identity. Diluted or undiluted DPs were inoculated in hemoculture bottles to assess sterility.

\section{Quality controls: viability and immunophenotype}

Quality controls were performed on the final DPs and their intermediate products in GMP conditions, using different methods, instruments and reagents according to the SOPs of each laboratory, and are therefore only briefly summarized below. All methods were validated and applied the same specifications as those for ATMP batch release, as established by each cell factory [19]. The methods and specifications are indicated in Tables 2, 3, and 4.

Viability was measured either by Trypan Blue (Sigma AldrichMerck, Darmstadt, Germany) dye exclusion and counting in a hemocytometer, or by staining with propidium iodide (PI; Sigma AldrichMerck or BD Biosciences, San Jose, CA) or 7-aminoactinomycin D (7$A A D$; BD Biosciences), followed by standard flow cytometry evaluation. Immunophenotypes were identified by staining with specific fluorescently labeled antibodies and flow cytometry analysis.

\section{Quality controls: potency assays}

Potency assays were those known for the type of cell product: immunosuppression and proliferation/differentiation for mesenchymal stromal cells (MSCs), cytotoxicity for $\mathrm{T}$ cell-derived products and $\mathrm{T}$ cell activation potential for dendritic cells (DCs). Cytotoxicity assays were performed using different methods according to each cell factory's internal protocols: (1) calcein-AM (Sigma Aldrich-Merck) loading of target cells and calcein release measured in an automated plate reader after 4-h coincubation of targets with effector cells (FLUOstar Optima, BMG LabTech), (2) carboxy fluorescein diacetate succinimidyl ester (CFSE; Molecular Probes, Eugene, OR) labeling of target cells followed by analysis of target cell death (apoptosis and necrosis) by PI/ annexin $\mathrm{V}$ staining, (3) labeling of target cells overnight with ${ }^{51}$ chromium $\left({ }^{51} \mathrm{Cr}, \mathrm{Na}_{2}{ }^{51} \mathrm{CrO}_{4}\right.$ solution; PerkinElmer, Boston, MA), followed by $5-\mathrm{h}{ }^{51} \mathrm{Cr}$ release assay and gamma counter readout, or (4) 3-[4,5dimethylthiazol-2-yl]-2,5 diphenyl tetrazolium bromide (MTT) assay (Promega, Medison, WI), as previously described [20].

Immunosuppression assays were performed by coincubation of ATMPs with peripheral blood mononuclear cells (PBMCs) stimulated with phytohemagglutinin (PHA; Sigma Aldrich-Merck). Proliferation was measured with either CFSE and flow cytometry or ${ }^{3} \mathrm{H}$-thymidine [21.22]. Proliferation was measured either by cell counting or capacity to form colonies in vitro $[23,24]$.

To measure the capacity of DCs to activate lymphocytes, mixed lymphocyte reactions (MLRs) were set up. Briefly, PBMCs (responder cells) were co-cultured with irradiated or mitomycin-C-treated stimulating $(\mathrm{S})$ cells at different ratios. S cells were DCs, autologous PBMCs (for auto-MLR, negative control), or allogeneic PBMCs (for allo-MLR, positive control). After 5 days of culture, proliferation was assessed using MTS reagent (3-(4,5-dimethylthiazol-2-yl)-5-(3-carboxymethoxyphenyl)-2-(4-sulfophenyl)-2H-tetrazolium, inner salt; Promega, Milano, Italy) and measured by absorbance at $540 \mathrm{~nm}$ [25]. Alternatively, proliferation was measured using an $18-\mathrm{h}{ }^{3} \mathrm{H}$-thymidine (PerkinElmer) incorporation assay [25].

IFN $\gamma$ production was measured by ELISPOT procedures. Briefly ATMPs were seeded on 96-well multiscreen filter plates coated with IFN $\gamma$ antibody (Mabtech, Nacka, Sweden), in the absence or presence of relevant peptide mixes. After incubation for $24 \mathrm{~h}$, plates were processed according to standard procedures, and spot-forming units (SFU) were counted using an ELISPOT reader (Bioline, Torino, Italy) [22]. The osteogenic differentiation potential of bone marrow (BM)-derived MSCs in 2D monolayers or 3D scaffolds in vitro, as well as in vivo ectopic bone formation, was assessed as described previously [26].

\section{Karyotyping}

Karyotyping was performed on an aliquot of cells by Q- or G-banding according to SOPs of the local Clinical Genetics Laboratories [27]. 
Table 1

ATMPs manufactured by Plagencell Network cell factories and major clinical trials

\begin{tabular}{|c|c|c|c|c|c|c|c|}
\hline $\begin{array}{l}\text { ATMP cell type } \\
\text { (abbreviation) }\end{array}$ & ATMP (abbreviation) & Manufacturer $^{\mathrm{a}}$ & Study Identification & Use & Phase & $\begin{array}{l}\text { No. of batches produced } \\
\text { for trials (including } \\
\text { compassionate use) }\end{array}$ & Reference \\
\hline \multirow{22}{*}{$\begin{array}{l}\text { Mesenchymal } \\
\text { stromal cells } \\
\text { (MSCs) }\end{array}$} & \multirow[t]{12}{*}{ Bone marrow-derived (BM-MSC) } & $\mathrm{CF} 1+\mathrm{CF} 2$ & NCT01764100 & Allogeneic & $\mathrm{I} / \mathrm{II}$ & 73 & \multirow{12}{*}{$\begin{array}{l}{[33]} \\
{[31,37]} \\
{[31]} \\
{[30]}\end{array}$} \\
\hline & & CF1 & NCT02012153 & Autologous & I & 6 & \\
\hline & & CF1 & NCT01854957 & Autologous & $\mathrm{I} / \mathrm{II}$ & 35 & \\
\hline & & CF1 & NCT02260375 & Allogeneic & $\mathrm{I} / \mathrm{II}$ & 4 & \\
\hline & & CF1 & NCT02565459 & Allogeneic & $\mathrm{I} / \mathrm{II}$ & 5 & \\
\hline & & CF1 & $\begin{array}{l}\text { EudraCT No. 2016- } \\
\quad 004804-77\end{array}$ & Autologous & I & 7 & \\
\hline & & CF3 & $\begin{array}{l}\text { EudraCT No. 2019- } \\
\quad 002749-40\end{array}$ & Autologous & IIb & 1 & \\
\hline & & CF3 & NCT04759105 & Autologous & IIb & 9 & \\
\hline & & CF3 & NCT01824121 & Autologous & $\mathrm{I}$ & 5 & \\
\hline & & CF3 & NCT02065167 & Autologous & II & 3 & \\
\hline & & CF5 & $\begin{array}{l}\text { EudraCT No. 2021- } \\
\quad 004755-17\end{array}$ & Allogeneic & $\mathrm{I} / \mathrm{II}$ & 44 & \\
\hline & & CF5 & NA & Allogeneic & NA & 18 & \\
\hline & Umbilical cord-derived (UCM) & CF1 & NCT02032446 & Allogeneic & $\mathrm{I} / \mathrm{II}$ & 18 & \multirow[t]{3}{*}{ Submitted } \\
\hline & $\begin{array}{l}\mathrm{CD}^{2} 62^{+} \text {selected BM stromal cells } \\
\text { (ORBCEL-M) }\end{array}$ & CF1 & NCT02585622 & Autologous & I & 3 & \\
\hline & \multirow[t]{2}{*}{$\begin{array}{l}\text { bone marrow-derived (BM- } \\
\text { MSC) + biomaterial }\end{array}$} & CF3 & NCT03325504 & Autologous & III & 5 & \\
\hline & & CF3 & NCT01842477 & Autologous & II & 7 & \multirow[t]{7}{*}[45]{} \\
\hline & \multirow[t]{3}{*}{ Cord blood-derived (CB-MSC) } & CF3 & NCT04034316 & Allogeneic & II & 11 & \\
\hline & & CF3 & $\begin{array}{l}\text { EudraCT No. 2011- } \\
\text { 001387-21 }\end{array}$ & Allogeneic & I & 11 & \\
\hline & & CF3 & $\begin{array}{l}\text { Eudra CT 2020-001577- } \\
\quad 70\end{array}$ & Allogeneic & $\mathrm{I} / \mathrm{II} \mathrm{a}$ & 3 & \\
\hline & Adipose tissue-derived (AD-MSC) & CF3 & $\begin{array}{l}\text { EudraCT No. 2020- } \\
\quad \text { 005336-29 }\end{array}$ & Autologous & II & $\begin{array}{l}\text { Clinical trial under sub- } \\
\text { mission to competent } \\
\text { authorities }\end{array}$ & \\
\hline & Adipose tissue-derived (AD-MSC) & CF4 & $\begin{array}{l}\text { Documents in } \\
\text { preparation }\end{array}$ & Allogeneic & I & 1 & \\
\hline & $\begin{array}{l}\text { AD-MSC loaded with Paclitaxel } \\
\text { (PacliMES) }\end{array}$ & CF4 & $\begin{array}{l}\text { EudraCT No. } 2020 \\
-005928-11\end{array}$ & Allogeneic & I & 1 & \\
\hline \multirow[t]{8}{*}{$\begin{array}{l}\text { Polyclonal T } \\
\text { cells }\end{array}$} & $\begin{array}{l}\text { Blinatumomab-expanded T cells } \\
\text { (BET) }\end{array}$ & CF1 & NCT03823365 & Autologous & I & 15 & \\
\hline & \multirow[t]{3}{*}{ Cytokine-induced killer (CIK) cells } & $\mathrm{CF} 1+\mathrm{CF} 2$ & NCT01186809 & Allogeneic & $\mathrm{II} / \mathrm{A}$ & 110 & [32] \\
\hline & & CF1 & ISS 64499-PRE21-848 & Allogeneic & I & 11 & \multirow[t]{4}{*}[42]{} \\
\hline & & CF1 & NCT03821519 & Allogeneic & $\mathrm{I} / \mathrm{II}$ & 8 & \\
\hline & Anti-leukemia cytotoxic T cells (CTL) & CF5 & $\begin{array}{l}\text { EudraCT No. 2019- } \\
\quad 003362-41\end{array}$ & Allogeneic & $\mathrm{I} / \mathrm{II}$ & 24 & \\
\hline & $\begin{array}{l}\text { Adenovirus-specific T lymphocytes } \\
\text { (LTC-ADV) }\end{array}$ & CF5 & $\begin{array}{l}\text { EudraCT No. 2008- } \\
\quad 000523-25\end{array}$ & Allogeneic or autologous & $\mathrm{I} / \mathrm{II}$ & 5 & \\
\hline & $\begin{array}{l}\text { Epstein-Barr virus-specific CTL } \\
\quad(\text { CTL-EBV) }\end{array}$ & CF5 & CE n. $15 / 97$ & Allogeneic or autologous & $\mathrm{I} / \mathrm{II}$ & 43 & \multirow[t]{2}{*}[35,39]{} \\
\hline & $\begin{array}{l}\text { Cytomegalovirus-specific T lympho- } \\
\text { cytes (LTC-CMV) }\end{array}$ & CF5 & NA & Allogeneic or autologous & NA & 9 & \\
\hline \multirow{2}{*}{$\begin{array}{l}\text { Gene modified } \\
\text { polyclonal T } \\
\text { cells }\end{array}$} & $\begin{array}{l}\text { CAR-CD19 gene-modified CIK (CAR- } \\
\text { CIK-CD19) }\end{array}$ & $\mathrm{CF} 1+\mathrm{CF} 2$ & NCT03389035 & Allogeneic & $\mathrm{I} / \mathrm{II}$ & 35 & \multirow[t]{4}{*}{ [38] } \\
\hline & & $\mathrm{CF} 1+\mathrm{CF} 2$ & $\begin{array}{l}\text { EudraCT No. 2020- } \\
\quad 005025-85\end{array}$ & $\begin{array}{r}\text { Allogeneic or } \\
\text { Autologous }\end{array}$ & II & 1 & \\
\hline \multirow[t]{3}{*}{$\begin{array}{l}\text { Dendritic cells } \\
\quad \text { (DCs) }\end{array}$} & $\begin{array}{l}\text { Autologous GSC a-loaded mature } \\
\text { DCs (DENDR STEM) }\end{array}$ & CF4 & $\begin{array}{l}\text { EudraCT No. 2013- } \\
\quad 002100-13\end{array}$ & Autologous & I & 2 & \\
\hline & $\begin{array}{l}\text { Tumor lysate-loaded mature DCs } \\
\text { (DENDR-1) }\end{array}$ & CF4 & $\begin{array}{l}\text { EudraCT No. 2008- } \\
\quad \text { 005035-15 }\end{array}$ & Autologous & $\mathrm{I} / \mathrm{II}$ & 65 & \\
\hline & $\begin{array}{l}\text { Tumor lysate-loaded mature DCs } \\
\text { (DENDR-2) }\end{array}$ & CF4 & $\begin{array}{l}\text { EudraCT No. 2008- } \\
\quad 005038-62\end{array}$ & Autologous & I & 40 & \multirow[t]{3}{*}{ [36] } \\
\hline \multirow[t]{2}{*}{ Stem cells } & $\begin{array}{l}\text { Human fetal neural stem precursor } \\
\text { cells (hNPC-DP) }\end{array}$ & CF2 & $\begin{array}{l}\text { EudraCT No. 2016- } \\
\text { 002020-86 }\end{array}$ & Allogeneic & I & 15 & \\
\hline & $\begin{array}{l}\text { BM-derived CD133 } 3^{+} \text {stem cells (BM- } \\
\text { CD133-SC) }\end{array}$ & $\mathrm{CF} 2$ & $\begin{array}{l}\text { EudraCT No. 2012- } \\
\quad 005267-27\end{array}$ & Autologous & I & 18 & \\
\hline
\end{tabular}

a CF1, Center of Cellular Therapy “G. Lanzani”, ASST Papa Giovanni XXIII, Bergamo; CF2, Laboratory of Cellular and Gene Therapy “S. Verri”, ASST San Gerardo, Monza; CF3, Laboratorio di Medicina Rigenerativa - Cell Factory, Fondazione IRCCS Ca' Granda Ospedale Maggiore Policlinico, Milan; CF4, Cell Therapy Production Unit UPTC, IRCCS Istituto Neurologico Carlo Besta, Milan; CF5, UOSD Cell Factory, Fondazione IRCCS Policlinico San Matteo, Pavia. NA, not applicable

\section{Microbiological contamination}

Endotoxin levels were measured by gel clot assay or the kinetic chromogenic method performed in-house (LAL-PTS system; Charles River, Wilmington, MA) or by Eurofins Biolab (Vimodrone, MI, Italy) according to Ph.Eur.2.6.14. Possible contamination with aerobic and anaerobic microbes was measured by hemoculture methods, using BactAlert (Biomerieux) or BACTEC (BD Diagnostics) according to the manufacturers' instructions and Ph.Eur.2.6.27. Mycoplasma contamination was measured using the culture method or by polymerase 
Stability studies on cryopreserved final DPs and data at longest time point

\begin{tabular}{|c|c|c|c|c|c|c|c|c|c|c|}
\hline $\begin{array}{l}\text { ATMP } \\
\text { (manufacturer) }\end{array}$ & Excipients & Primary container & Freezing method & Storage & $\begin{array}{l}\text { Shelf life assigned } \\
\text { (no. batches tested) }\end{array}$ & $\begin{array}{l}\text { QC test performed } \\
\text { to assign shelf life }\end{array}$ & Test method & Test specifications & $\begin{array}{l}\text { QC test result before } \\
\text { freezing }\end{array}$ & $\begin{array}{l}\text { QC test result at } \\
\text { latest time point } \\
\text { tested }\end{array}$ \\
\hline \multirow{9}{*}{$\begin{array}{l}\text { BM-MSC +UCM } \\
\text { (CF1) }\end{array}$} & \multirow{9}{*}{$\begin{array}{l}\text { Human plasma } \\
\qquad \mathrm{AB}+10 \% \text { DMSO }\end{array}$} & \multirow{9}{*}{$\begin{array}{l}\text { CryoMACS Miltenyi } \\
\text { infusion bag }\end{array}$} & \multirow{9}{*}{$\begin{array}{l}\text { Controlled-rate } \\
\text { freezer }\end{array}$} & \multirow{9}{*}{$\leq-150^{\circ} \mathrm{C}$} & \multirow{9}{*}{8 years $(n=3)$} & Viability & Trypan blue & $\geq 80 \%$ viability & $94.5 \% \pm 3.2 \%$ & $89.3 \% \pm 3.3 \%$ \\
\hline & & & & & & Immunophenotype & Cytofluorimetry & $\mathrm{CD} 73^{+} \geq 90 \%$ & $97.4 \% \pm 1.2 \%$ & $99.6 \% \pm 0.2 \%$ \\
\hline & & & & & & & & $\mathrm{CD} 0^{+} \geq 90 \%$ & $99.3 \% \pm 0.5 \%$ & $99.8 \% \pm 0.3 \%$ \\
\hline & & & & & & & & $\mathrm{CD} 105^{-} \geq 90 \%$ & $97.1 \% \pm 0.6 \%$ & $98.9 \% \pm 1.4 \%$ \\
\hline & & & & & & Immunosuppression & Cytofluorimetry & $\begin{array}{l}\geq 30 \% \text { PBMC prolifera- } \\
\text { tion inhibition at } \mathrm{E}: \mathrm{T} \\
\text { ratio } 10: 1\end{array}$ & $\mathrm{ND}$ & $64.8 \% \pm 3.4 \%$ \\
\hline & & & & & & Karyotype & QFQ-banding & $\begin{array}{l}\text { No metaphases or no } \\
\text { chromosomic aberra- } \\
\text { tion or non-clonal } \\
\text { chromosomic aberra- } \\
\text { tions in } \leq 10 \% \text { of meta- } \\
\text { phases [27] }\end{array}$ & Compliant & Compliant \\
\hline & & & & & & Sterility & Bact/Alert & Sterile & Sterile & Sterile \\
\hline & & & & & & Mycoplasma & Culture & Absence & Absent & Absent \\
\hline & & & & & & Endotoxin Level & Chromokinetic & $<7 \mathrm{EU} / \mathrm{mL}$ & $<3.5 \mathrm{EU} / \mathrm{mL}$ & $<3.5 \mathrm{EU} / \mathrm{mL}$ \\
\hline \multirow[t]{4}{*}{ BM-MSC (CF2) } & \multirow{4}{*}{$\begin{array}{l}\text { HSA } 20 \%+10 \% \\
\text { ACD + } 10 \% \text { DMSO }\end{array}$} & \multirow[t]{4}{*}{ Nalgene cryovial } & \multirow{4}{*}{$\begin{array}{l}\text { Controlled-rate } \\
\text { freezer }\end{array}$} & \multirow{4}{*}{$\leq-150^{\circ} \mathrm{C}$} & \multirow{4}{*}{49 months (n=1) } & Viability & Cytofluorimetry & $>70 \%$ viability & $87.7 \%$ & $82.3 \%$ \\
\hline & & & & & & Immunophenotype & Cytofluorimetry & $\mathrm{CD} 3^{+} \geq 70 \%$ & $99.3 \%$ & $99.5 \%$ \\
\hline & & & & & & & & $\mathrm{CD} 0^{+} \geq 70 \%$ & $99.7 \%$ & $99.6 \%$ \\
\hline & & & & & & & & $\mathrm{CD} 105^{-} \geq 70 \%$ & $99.9 \%$ & $99.7 \%$ \\
\hline \multirow[t]{7}{*}{ BM-MSC (CF5) } & \multirow{7}{*}{$\begin{array}{l}\mathrm{NaCl} 0.9 \%+5 \% \mathrm{HSA}+ \\
\quad 10 \% \text { DMSO }\end{array}$} & \multirow{7}{*}{ Nalgene cryovial } & \multirow{7}{*}{$\begin{array}{l}\text { Nalgene controlled- } \\
\text { rate manual freez- } \\
\text { ing system }\end{array}$} & \multirow{7}{*}{$\leq-150^{\circ} \mathrm{C}$} & \multirow[t]{7}{*}{6 years $(n=3)$} & Viability & Trypan blue & $\geq 90 \%$ viability & $96.0 \% \pm 1.5 \%$ & $93.0 \% \pm 3.0 \%$ \\
\hline & & & & & & Immunophenotype & Cytofluorimetry & $\mathrm{CD}^{-} 3^{+} \geq 85 \%$ & $98.0 \% \pm 0.7 \%$ & $94.0 \% \pm 6.0 \%$ \\
\hline & & & & & & & & $\mathrm{CD} 0^{+} \geq 85 \%$ & $99.0 \% \pm 0.5 \%$ & $96.0 \% \pm 5.0 \%$ \\
\hline & & & & & & & & $\mathrm{CD} 105^{+} \geq 85 \%$ & $96.0 \% \pm 0.9 \%$ & $94.0 \% \pm 8.0 \%$ \\
\hline & & & & & & $\begin{array}{l}\text { Differentiation } \\
\text { potential }\end{array}$ & $\begin{array}{l}\text { Cell culture and spe- } \\
\text { cific staining }\end{array}$ & $\begin{array}{l}\text { Differentiation into adi- } \\
\text { pocytes and osteocyte }\end{array}$ & Yes & Yes \\
\hline & & & & & & Immunosuppression & ${ }^{3} \mathrm{H} \mathrm{TdR}$ & $\begin{array}{l}\geq 30 \% \text { inhibition at } \mathrm{E}: \mathrm{T} \\
\text { ratio } 1: 2\end{array}$ & $65.0 \% \pm 14.0 \%$ & $71.0 \% \pm 9.0 \%$ \\
\hline & & & & & & Sterility & Bactec & Sterile & Sterile & Sterile \\
\hline \multirow{7}{*}{$\begin{array}{l}\text { ORBCEL-M } \\
\text { (CF1) }\end{array}$} & \multirow[t]{7}{*}{ Cryostor CS10 } & \multirow{7}{*}{$\begin{array}{l}\text { CryoMACS Miltenyi } \\
\text { infusion bag }\end{array}$} & \multirow{7}{*}{$\begin{array}{l}\text { Controlled-rate } \\
\text { freezer }\end{array}$} & \multirow[t]{7}{*}{$\leq-150^{\circ} \mathrm{C}$} & 45 months (n=3) & Viability & Trypan Blue & $\geq 70 \%$ viability & $85.3 \% \pm 3.5 \%$ & $90.1 \% \pm 2.9 \%$ \\
\hline & & & & & & Immunophenotype & Cytofluorimetry & $\mathrm{CD} 73^{+} \geq 95 \%$ & $99.9 \% \pm 0.1 \%$ & $99.8 \% \pm 0.1 \%$ \\
\hline & & & & & & & & $\mathrm{CD}^{+} 0^{+} \geq 95 \%$ & $99.9 \% \pm 0.1 \%$ & $99.9 \% \pm 0.1 \%$ \\
\hline & & & & & & & & $\mathrm{CD} 105^{-} \geq 95 \%$ & $99.6 \% \pm 0.5 \%$ & $98.3 \% \pm 2.3 \%$ \\
\hline & & & & & & Sterility & Bact/Alert & Sterile & Sterile & Sterile \\
\hline & & & & & & Mycoplasma & Culture & Absence & Absent & Absent \\
\hline & & & & & & Endotoxin Level & Chromokinetic & $<10 \mathrm{EU} / \mathrm{mL}$ & $\leq 5 \mathrm{EU} / \mathrm{mL}$ & $\leq 5 \mathrm{EU} / \mathrm{mL}$ \\
\hline CB-MSC (CF3) & $\mathrm{NaCl} 0.9 \%+10 \%$ & CryoMACS Miltenyi & Controlled-rate & $\leq-150^{\circ} \mathrm{C}$ & 57 months $(\mathrm{n}=3)$ & Viability & Cytofluorimetry & $\geq 80 \%$ viability & $88.5 \% \pm 6.2 \%$ & $87.6 \% \pm 3.7$ \\
\hline & $\mathrm{HSA}+10 \%$ DMSO & freezing bag & freezer & & & Immunophenotype & Cytofluorimetry & $\begin{array}{l}\mathrm{CD} 45^{-} \mathrm{CD}^{\circ} 0^{+} \mathrm{CD} 105^{+} \\
\geq 90 \%\end{array}$ & $92.8 \% \pm 1.4 \%$ & $98.3 \% \pm 1.2 \%$ \\
\hline & & & & & & $\begin{array}{l}\text { Proliferation } \\
\text { Potential }\end{array}$ & Gentian Violet & Colony formation & $52.0 \pm 22.4$ & $19.0 \pm 15.8$ \\
\hline & & & & & & & Fold expansion & $\geq 2$ fold & $24.4 \pm 7.1$ & $6.8 \pm 4.3$ \\
\hline & & & & & & Karyotype & G-banding & $46, \mathrm{XX}$ or $46, \mathrm{XY}$ & Compliant & Compliant \\
\hline & & & & & & Sterility & Bact/Alert & Sterile & Sterile & Sterile \\
\hline AD-MSC (CF4) & $\mathrm{NaCl} 0.9 \%+5 \%$ & Nalgene cryovial & Controlled-rate & $\leq-150^{\circ} \mathrm{C}$ & 1 years $(n=1)$ & Viability & Trypan blue & $\geq 80 \%$ viability & $96.1 \%$ & $92.9 \%$ \\
\hline & HSA + 10\% DMSO & & freezer & & & Immunophenotype & Cytofluorimetry & $\mathrm{CD}^{2} 3^{+} \geq 80 \%$ & $98.6 \%$ & $97.6 \%$ \\
\hline & & & & & & & & $\mathrm{CD} 0^{+} \geq 80 \%$ & $94.5 \%$ & $94.9 \%$ \\
\hline & & & & & & & & $\mathrm{CD} 105^{+} \geq 80 \%$ & $90.5 \%$ & $91.0 \%$ \\
\hline & & & & & & Sterility & Bact/Alert & Sterile & Sterile & Sterile \\
\hline & & & & & & Mycoplasma & PCR & Absence & Absent & Absent \\
\hline & & & & & & Endotoxin Level & Gel Clot & $<117 \mathrm{EU} / \mathrm{mL}$ & $\leq 0.5 \mathrm{EU} / \mathrm{mL}$ & $\leq 0.5 \mathrm{EU} / \mathrm{mL}$ \\
\hline PacliMES (CF4) & & Nalgene cryovial & & $\leq-150^{\circ} \mathrm{C}$ & 1 years $(n=3)$ & Viability & Trypan blue & $\geq 80 \%$ viability & $91.0 \% \pm 0.5 \%$ & $90.5 \% \pm 2.2 \%$ \\
\hline
\end{tabular}




\begin{tabular}{|c|c|c|c|c|c|c|c|c|c|c|}
\hline $\begin{array}{l}\text { ATMP } \\
\text { (manufacturer) }\end{array}$ & Excipients & Primary container & Freezing method & Storage & $\begin{array}{l}\text { Shelf life assigned } \\
\text { (no. batches tested) }\end{array}$ & $\begin{array}{l}\text { QC test performed } \\
\text { to assign shelf life }\end{array}$ & Test method & Test specifications & $\begin{array}{l}\text { QC test result before } \\
\text { freezing }\end{array}$ & $\begin{array}{l}\text { QC test result at } \\
\text { latest time point } \\
\text { tested }\end{array}$ \\
\hline & $\mathrm{NaCl} 0.9 \%+5 \%$ & & Controlled-rate & & & Immunophenotype & Cytofluorimetry & $\mathrm{CD} 73^{+} \geq 80 \%$ & $98.4 \% \pm 0.4 \%$ & $99.3 \% \pm 0.9 \%$ \\
\hline & HSA + 10\% DMSO & & freezer & & & & & $\mathrm{CD}^{+} 0^{+} \geq 80 \%$ & $93.9 \% \pm 3.9 \%$ & $94.1 \% \pm 4.0 \%$ \\
\hline & & & & & & & & $\mathrm{CD} 105^{+} \geq 80 \%$ & $91.5 \% \pm 7.7 \%$ & $83.4 \% \pm 1.8 \%$ \\
\hline & & & & & & Paclitaxel content & $\begin{array}{l}\text { HPLC/Mass } \\
\text { spectrometry }\end{array}$ & $\geq 0.05 \mathrm{pg} / \mathrm{cell}$ & $0.5 \pm 0.2$ & $0.5 \pm 0.2$ \\
\hline & & & & & & Cytotoxicity & MTT & $\begin{array}{l}\geq 13.5 \mathrm{ng} / \mathrm{mL} \text { (Paclitaxel } \\
\text { equivalent } \\
\text { concentration) }\end{array}$ & $13.7 \pm 0.0$ & $13 \pm 0.0$ \\
\hline & & & & & & Sterility & Bact/Alert & Sterile & Sterile & Sterile \\
\hline & & & & & & Mycoplasma & PCR & Absence & Absent & Absent \\
\hline & & & & & & Endotoxin Level & Gel Clot & $<117 \mathrm{EU} / \mathrm{mL}$ & $\leq 0.5 \mathrm{EU} / \mathrm{mL}$ & $\leq 0.5 \mathrm{EU} / \mathrm{mL}$ \\
\hline \multirow[t]{2}{*}{ BET (CF1) } & Human plasma & CryoMACS Miltenyi & Controlled-rate & $\leq-150^{\circ} \mathrm{C}$ & 2 years $(n=1)$ & Viability & Cytofluorimetry & $\geq 80 \%$ viability & $80.7 \%$ & $94.5 \%$ \\
\hline & $\mathrm{AB}+10 \% \mathrm{DMSO}$ & infusion bag & freezer & & & Immunophenotype & Cytofluorimetry & $\mathrm{CD}^{+} \geq 90 \%$ & $84.5 \%$ & $85.7 \%$ \\
\hline \multirow[t]{3}{*}{ CIK (CF1) } & Human plasma & Hemofreeze bag & Controlled-rate & $\leq-150^{\circ} \mathrm{C}$ & 7 years $(n=1)$ & Viability & Cytofluorimetry & $\geq 80 \%$ viability & $96.9 \%$ & $98 \%$ \\
\hline & $\mathrm{AB}+10 \%$ DMSO & Fresenius & freezer & & & Immunophenotype & Cytofluorimetry & $\mathrm{CD}^{+} \mathrm{CD}^{2} 6^{+} \geq 40 \%$ & $51.3 \%$ & $48.8 \%$ \\
\hline & & & & & & Cytotoxicity & Calcein-AM release & $\begin{array}{l}\geq 10 \% \text { lysis at } \mathrm{E}: \mathrm{T} \text { ratio } \\
30: 1\end{array}$ & ND & $45.1 \%$ \\
\hline \multirow[t]{2}{*}{ CIK (CF2) } & HSA $20 \%+10 \%$ & SAFE2 Cell cryobag & Controlled-rate & $\leq-150^{\circ} \mathrm{C}$ & 2 years $(n=3)$ & Viability & Cytofluorimetry & $\geq 70 \%$ viability & $79.2 \% \pm 5.8 \%$ & $91.9 \% \pm 3.6 \%$ \\
\hline & DMSO + 10\% ACD & Gobbi Frattini & freezer & & & Immunophenotype & Cytofluorimetry & $\mathrm{CD} 3^{+} \mathrm{CD} 6^{+} \geq 40 \%$ & $45 \% \pm 7.1 \%$ & $44.4 \% \pm 2.4 \%$ \\
\hline \multirow[t]{4}{*}{ CTL (CF5) } & $\mathrm{NaCl} 0.9 \%+5 \% \mathrm{HSA}^{+}$ & Nalgene cryovial & Nalgene controlled- & $\leq-150^{\circ} \mathrm{C}$ & 8 years $(n=3)$ & Viability & Trypan blue & $\geq 80 \%$ viability & $94 \% \pm 5 \%$ & $88 \% \pm 3 \%$ \\
\hline & 10\% DMSO & & $\begin{array}{l}\text { rate manual freez- } \\
\text { ing system }\end{array}$ & & & Immunophenotype & Cytofluorimetry & $\begin{array}{l}\mathrm{CD}^{+} / \mathrm{CD}^{-} \mathrm{CD}^{\mathrm{C}} 6^{+} \text {value } \\
\text { at freezing } \pm 10 \%\end{array}$ & $98 \% \pm 2 \%$ & $98 \% \pm 2 \%$ \\
\hline & & & & & & Cytotoxicity & ${ }^{51} \mathrm{Cr}$ release & $\begin{array}{l}\text { Cytotoxicity value at } \\
\text { freezing } \pm 10 \%\end{array}$ & $25 \% \pm 15 \%$ & $27 \% \pm 7 \%$ \\
\hline & & & & & & Sterility & Bactec & Sterile & Sterile & Sterile \\
\hline \multirow[t]{4}{*}{ LTC-ADV (CF5) } & $\mathrm{NaCl} 0.9 \%+5 \%$ & Nalgene cryovial & Nalgene controlled- & $\leq-150^{\circ} \mathrm{C}$ & 13.5 years $(n=5)$ & Viability & Trypan blue & $\geq 80 \%$ viability & $100 \% \pm 0 \%$ & $96 \% \pm 0.5 \%$ \\
\hline & $\mathrm{HSA}+10 \% \mathrm{DMSO}$ & & $\begin{array}{l}\text { rate manual freez- } \\
\text { ing system }\end{array}$ & & & Immunophenotype & Cytofluorimetry & $\begin{array}{l}\mathrm{CD}^{+} / \mathrm{CD}^{-} \mathrm{CD}^{-} 6^{+} \text {value } \\
\text { at freezing } \pm 10 \%\end{array}$ & $98 \% \pm 2 \%$ & $99 \% \pm 1 \%$ \\
\hline & & & & & & Cytotoxicity & $\mathrm{Cr}^{51}$ release & $\begin{array}{l}\text { Cytotoxicity }>25 \% \text { at E:T } \\
\text { ratio } 20: 1\end{array}$ & $62 \% \pm 20 \%$ & $45 \% \pm 18 \%$ \\
\hline & & & & & & Sterility & Bactec & Sterile & Sterile & Sterile \\
\hline \multirow[t]{4}{*}{ CTL-EBV (CF5) } & $\mathrm{NaCl} 0.9 \%+5 \%$ & Nalgene cryovial & Nalgene controlled- & $\leq-150^{\circ} \mathrm{C}$ & 4 years $(n=3)$ & Viability & Trypan blue & $\geq 80 \%$ viability & $100 \% \pm 0 \%$ & $96 \% \pm 0.5 \%$ \\
\hline & $\mathrm{HSA}+10 \% \mathrm{DMSO}$ & & $\begin{array}{l}\text { rate manual freez- } \\
\text { ing system }\end{array}$ & & & Immunophenotype & Cytofluorimetry & $\begin{array}{l}\mathrm{CD}^{+} / \mathrm{CD}^{-} \mathrm{CD}^{-} 6^{+} \text {value } \\
\text { at freezing } \pm 10 \%\end{array}$ & $98 \% \pm 2 \%$ & $99 \% \pm 1 \%$ \\
\hline & & & & & & Cytotoxicity & $\mathrm{Cr}^{51}$ release & $\begin{array}{l}\text { Lysis > 25\% at E:T ratio } \\
\text { 20:1 }\end{array}$ & $62 \% \pm 20 \%$ & $45 \% \pm 18 \%$ \\
\hline & & & & & & Sterility & Bactec & Sterile & Sterile & Sterile \\
\hline \multirow[t]{4}{*}{ LTC-CMV (CF5) } & $\mathrm{NaCl} 0.9 \%+5 \%$ & Nalgene cryovial & Nalgene controlled- & $\leq-150^{\circ} \mathrm{C}$ & 2 years $(n=3)$ & Viability & Trypan blue & $\geq 80 \%$ viability & $100 \% \pm 0 \%$ & $85 \% \pm 5 \%$ \\
\hline & $\mathrm{HSA}+10 \% \mathrm{DMSO}$ & & $\begin{array}{l}\text { rate manual freez- } \\
\text { ing system }\end{array}$ & & & Immunophenotype & Cytofluorimetry & $\begin{array}{l}\mathrm{CD}^{+} / \mathrm{CD}^{-}{ }^{-} \mathrm{CD} 56^{+} \text {value } \\
\text { at freezing } \pm 10 \%\end{array}$ & $95 \% \pm 1.5 \%$ & $97 \% \pm 2 \%$ \\
\hline & & & & & & IFN- $\gamma$ production & ELISPOT assay & $\begin{array}{l}\text { Spot forming unit } / 10^{5} \\
\quad \text { cells }>25\end{array}$ & $473 \pm 236$ & $290 \pm 122$ \\
\hline & & & & & & Sterility & Bactec & Sterile & Sterile & Sterile \\
\hline \multirow{6}{*}{$\begin{array}{l}\text { CARCIK-CD19 } \\
\text { (CF1) }\end{array}$} & $0.9 \% \mathrm{NaCl}+11.2 \%$ & CryoMACS Miltenyi & Controlled-rate & $\leq-150^{\circ} \mathrm{C}$ & 1 year $(n=1)$ & Viability & Trypan blue & $\geq 70 \%$ viability & $94 \%$ & $82.8 \%$ \\
\hline & $\mathrm{HSA}+10 \%$ & double bags & freezer & & & Immunophenotype & Cytofluorimetry & $\mathrm{CD}^{+} \geq 90 \%$ & $98.5 \%$ & $98.6 \%$ \\
\hline & DMSO + 10\% ACD & & & & & & & $\mathrm{CD}^{+} 56^{+} \geq 30 \%$ & $57.7 \%$ & $57.7 \%$ \\
\hline & & & & & & & & $\mathrm{CAR}^{+} \geq 20 \%$ & $35.9 \%$ & $39.9 \%$ \\
\hline & & & & & & Cytotoxicity & Cytofluorimetry & $\geq 25 \%$ at E:T ratio 5:1 & $69.3 \%$ & $44.3 \%$ \\
\hline & & & & & & Sterility & Bact/Alert & Sterile & Sterile & Sterile \\
\hline \multirow{4}{*}{$\begin{array}{l}\text { CARCIK-CD19 } \\
\text { (CF2) }\end{array}$} & $0.9 \% \mathrm{NaCl}+11.2 \%$ & SAFE2 Cell cryobag & Controlled-rate & $\leq-150^{\circ} \mathrm{C}$ & 1 year $(n=2)$ & Viability & Trypan blue & $\geq 70 \%$ viability & $97.2 \% \pm 1.8 \%$ & $84.0 \% \pm 10.9 \%$ \\
\hline & $\mathrm{HSA}+10 \%$ & Gobbi Frattini & freezer & & & Immunophenotype & Cytofluorimetry & $\mathrm{CD}^{+} \geq 90 \%$ & $99.2 \% \pm 0.2 \%$ & $99.5 \% \pm 0.3 \%$ \\
\hline & DMSO + 10\% ACD & & & & & & & $\mathrm{CD}^{+} 56^{+} \geq 30 \%$ & $57.8 \% \pm 7.5 \%$ & $52.3 \% \pm 1.3 \%$ \\
\hline & & & & & & & & $\mathrm{CAR} \geq 20 \%$ & $56.5 \% \pm 13.6 \%$ & $44.8 \% \pm 14.0 \%$ \\
\hline
\end{tabular}




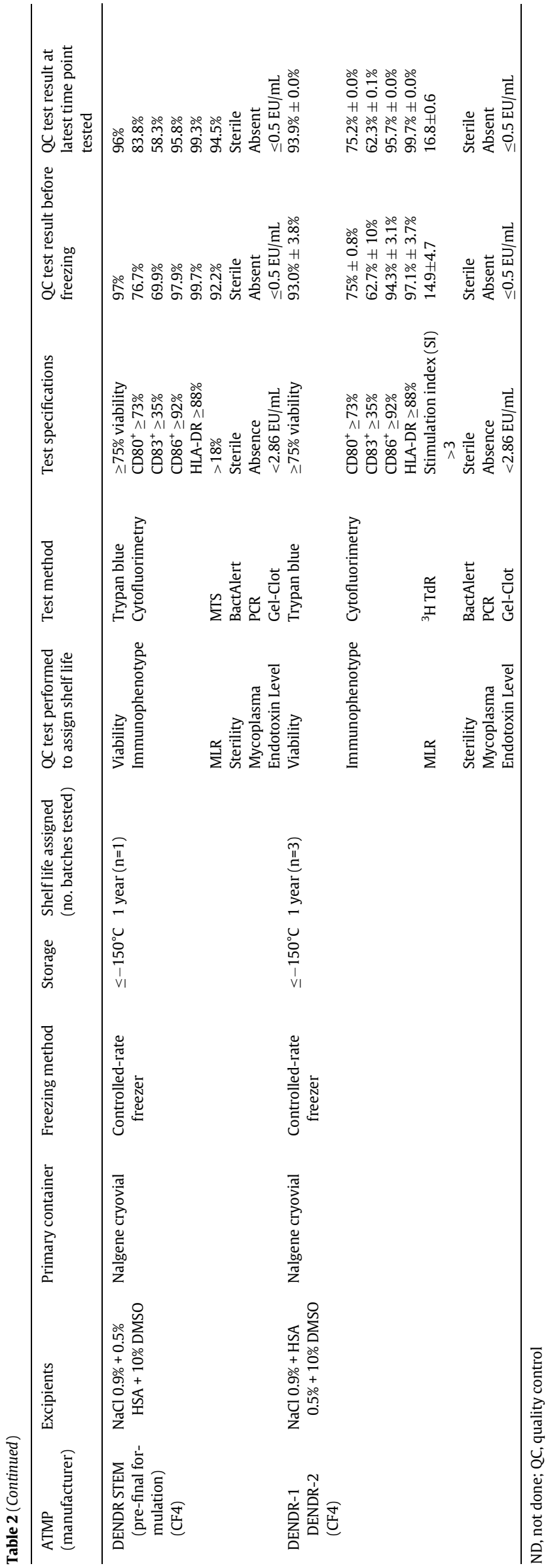

chain reaction (PCR), in all cases according to European Pharmacopeia (Ph.Eur.2.6.7; Ph Eur.2.6.21). Tests were carried out either in a GMP-qualified external laboratory (Eurofins Biolab) or in-house.

\section{Results}

The cell factory network and ATMPs produced for clinical use

The Plagencell cell factory network was created to optimize the development and production of innovative ATMPs for clinical use and is part of a wider collaboration between research institutes and hospitals in the Lombardy region (see Methods for details), which collaborate for the development of treatments for several devastating human diseases. The five cell factories are academic and have been approved for production and testing of experimental ATMPs for phase I/II clinical trials [28].

Table 1 lists the experimental ATMPs, clinical trials and numbers of DP batches that are the object of this report. The ATMPs belong to five different cell types as follows: (1) MSCs isolated from BM, umbilical cord (UC), adipose tissue (AD) and cord blood (CB). In one case, AD-derived MSCs were loaded with an anti-neoplastic agent (Paclitaxel). (2) Polyclonal T cells expanded from peripheral blood (PB) or $\mathrm{CB}$, including cytokine induced killer (CIK) cells, blinatumomab expanded T cells (BETs), cytotoxic T lymphocytes (CTLs) directed against Epstein-Barr virus (EBV) or leukemic cells, and $\mathrm{T}$ cell lines (LTCs) specific for cytomegalovirus (CMV) or adenovirus (ADV). (3) One gene-modified ATMP, produced by two cell factories, consists of CIK cells transduced with an anti-CD19 chimeric antigen receptor (CAR), using a nonviral transposon-based transfection method (CARCIK-CD19). (4) DCs starting from monocytes isolated from leukapheretic material and loaded with tumor cells or cell lysates. (5) Stem cells, including BM-derived $\mathrm{CD} 133^{+}$cells and neural precursor stem cells (NPCs), derived from fetal human brain (Table 1). Some ATMP types were made by more than one cell factory and entered stability studies in each. They were produced for 36 approved clinical trials, and overall 668 batches of cells have been produced in the context of these trials (Table 1 ).

\section{Stability studies performed on cryopreserved DPs}

Nineteen DPs produced (listed in Table 2) were cryopreserved to $<-150^{\circ} \mathrm{C}$ and stored in continuously monitored nitrogen vapor freezers. Because of the required cell volumes, treatment modalities and different practices, DPs were frozen in different types of primary containers (freezing bags or cryovials) and with different excipients, including DMSO added to human plasma or physiological solution containing different percentages of HSA and anticoagulant citrate dextrose $(A C D)$, or in preformulated freezing medium (Cryostor CS10) (Table 2). All ATMPs produced entered stability studies to assign an initial shelf life. The latter was extended once the results of the longer stability studies became available, so some ATMPs currently have a 7 - to 13.5 -year stability at $<-150^{\circ} \mathrm{C}$. Only the longest validated shelf lives are reported in Table 2 . The ATMPs more recently introduced have a shorter assigned stability due to the shorter period of testing or lack of clinical need to extend shelf life beyond 1 year.

The quality control methods performed for stability studies differed according to the ATMP and the cell factory (Table 2) but remained constant throughout each individual stability study. Usually, at least three batches of DPs produced in GMP and cryopreserved in the same concentrations, excipients and containers as those of the DPs for clinical use were tested in these studies at each time point, although in some cases, single batches were tested at different time points, following a matrixing approach.

The tests performed in stability studies included in all cases viability (by flow cytometry or Trypan Blue), identity and purity (immunophenotype). Acceptable viability was between $\geq 70 \%$ and $\geq 80 \%$ for 18 of 19 


\begin{tabular}{|c|c|c|c|c|c|c|c|c|c|}
\hline $\begin{array}{l}\text { ATMP } \\
\text { (manufacturer) }\end{array}$ & Excipients & Primary container & Storage & $\begin{array}{l}\text { Shelf life assigned ( } \mathrm{n} \\
{ }^{\circ} \text { batches tested) }\end{array}$ & $\begin{array}{l}\text { QC test performed } \\
\text { to assign shelf life }\end{array}$ & Test method & Test specifications & $\begin{array}{l}\text { QC test result before } \\
\text { freezing }\end{array}$ & $\begin{array}{l}\text { QC test result at } \\
\text { latest time point } \\
\text { tested }\end{array}$ \\
\hline \multirow[t]{9}{*}{$\begin{array}{l}\text { BM-MSC+ biomate- } \\
\text { rial (CF3) }\end{array}$} & $\mathrm{NaCl} 0.9 \%+5 \% \mathrm{HSA}$ & $\begin{array}{l}\text { Syringe with a } \\
\text { screwed luer lock } \\
\text { cap }\end{array}$ & $4-8^{\circ} \mathrm{C}$ & $\begin{array}{c}24 \mathrm{~h}(\mathrm{n}=10 \text { before } \\
\text { shipping, } \mathrm{n}=5 \text { at } \\
\text { latest time })\end{array}$ & Viability & Cytofluorimetry & $>80 \%$ viability & $98.7 \% \pm 0.6 \%$ & $93.9 \% \pm 0.7 \%$ \\
\hline & & & & & Immunophenotype & Cytofluorimetry & $\mathrm{CD} 73^{+} \geq 90 \%$ & $95.5 \% \pm 2.9 \%$ & $99.8 \% \pm 0.0 \%$ \\
\hline & & & & & & & $\mathrm{CD} 0^{+} \geq 90 \%$ & $97.7 \% \pm 2.2 \%$ & $99.8 \% \pm 0.2 \%$ \\
\hline & & & & & & & $\mathrm{CD} 105^{-} \geq 90 \%$ & $97.1 \% \pm 2.7 \%$ & $98.8 \% \pm 1.3 \%$ \\
\hline & & & & & & & $\mathrm{HLA} \mathrm{Cl} \mathrm{II} \leq 20 \%$ & $2.1 \% \pm 3.9 \%$ & $1.3 \% \pm 1.0 \%$ \\
\hline & & & & & $\begin{array}{l}\text { Proliferation } \\
\text { potential }\end{array}$ & $\begin{array}{l}\text { Crystal violet } \\
\text { staining }\end{array}$ & Cell adhesion to a 3D scaffold & [26] & \\
\hline & & & & & & Cell count & Fold Increase $\geq 2$ at day 6 in vitro & & \\
\hline & & & & & $\begin{array}{l}\text { Differentiation } \\
\text { potential }\end{array}$ & $\begin{array}{l}\text { Cell culture and spe- } \\
\text { cific staining [26] }\end{array}$ & $\begin{array}{l}\text { Ex vivo osteogenic differentiation com- } \\
\text { pared to controls }\end{array}$ & & \\
\hline & & & & & & $\begin{array}{l}\text { In vivo bone-forma- } \\
\text { tion assay }\end{array}$ & $\begin{array}{l}\text { Repair of induced calvaria defects after } 4 \\
\text { weeks }\end{array}$ & & \\
\hline \multirow[t]{5}{*}{ BM-MSC (CF3) } & $\mathrm{NaCl} 0.9 \%+5 \% \mathrm{HSA}$ & Syringe with a & $4-8^{\circ} \mathrm{C}$ & $24 \mathrm{~h}(\mathrm{n}=3)$ & Viability & Cytofluorimetry & $\geq 80 \%$ viability & $97.1 \% \pm 0.8 \%$ & $85.3 \% \pm 5.0$ \\
\hline & & screwed luer lock & & & Immunophenotype & Cytofluorimetry & $\mathrm{CD} 3^{+} \geq 90 \%$ & $99.9 \% \pm 0.0 \%$ & $99.8 \% \pm 0.3 \%$ \\
\hline & & cap & & & & & $\mathrm{CD}^{+} 0^{+} \geq 90 \%$ & $99.6 \% \pm 0.4 \%$ & $99.4 \% \pm 0.6 \%$ \\
\hline & & & & & & & $\mathrm{CD} 105^{-} \geq 90 \%$ & $99.8 \% \pm 0.2 \%$ & $99.7 \% \pm 0.4 \%$ \\
\hline & & & & & Sterility & Bact/Alert & Sterile & Sterile & Sterile \\
\hline \multirow[t]{6}{*}{ AD-MSC (CF3) } & Ringer lactate & Syringe with a & $2-8^{\circ} \mathrm{C}$ & $24 \mathrm{~h}(\mathrm{n}=3)$ & Viability & Cytofluorimetry & $\geq 80 \%$ viability & $84.1 \% \pm 0.3 \%$ & $86.4 \% \pm 2.9 \%$ \\
\hline & & screwed luer lock & & & Immunophenotype & Cytofluorimetry & $\mathrm{CD} 73^{+} \geq 80 \%$ & $98.9 \% \pm 0.8 \%$ & $99.7 \% \pm 0.3 \%$ \\
\hline & & cap & & & & & $\mathrm{CD} 0^{+} \geq 80 \%$ & $99.9 \% \pm 0.1 \%$ & $100.0 \% \pm 0.1 \%$ \\
\hline & & & & & & & $\mathrm{CD}_{105^{+}} \geq 80 \%$ & $99.4 \% \pm 0.5 \%$ & $98.5 \% \pm 1.7 \%$ \\
\hline & & & & & & & $\mathrm{CD} 166^{+} \geq 80 \%$ & $99.0 \% \pm 0.7 \%$ & $99.0 \% \pm 1.1 \%$ \\
\hline & & & & & Sterility & Bact/Alert & Sterile & Sterile & Sterile \\
\hline hNPC - DP (CF2) & $\begin{array}{l}\mathrm{NaCl} 0.9 \%+0.028 \% \\
\mathrm{HSA}\end{array}$ & $\begin{array}{l}\text { MACS GMP cell } \\
\text { expansion bag; } \\
\text { Miltenyi }\end{array}$ & $2-8^{\circ} \mathrm{C}$ & $4 \mathrm{~h}(\mathrm{n}=2)$ & Viability & Trypan Blue & $\geq 45 \%$ viability & $60.5 \% \pm 13.5 \%$ & $55.7 \% \pm 4.1 \%$ \\
\hline \multirow[t]{2}{*}{ BM-CD133-SC (CF2) } & $\mathrm{NaCl} 0.9 \%+5 \% \mathrm{HSA}$ & $\begin{array}{l}\text { 50-mL polypropyl- } \\
\text { ene tube }\end{array}$ & $4-8^{\circ} \mathrm{C}$ & $12 \mathrm{~h}(\mathrm{n}=3)$ & Viability & Trypan Blue & $\geq 80 \%$ viability & $92.1 \% \pm 4.3 \%$ & $92.4 \% \pm 1.2 \%$ \\
\hline & & & & & Immunophenotype & Cytofluorimetry & $\mathrm{CD}_{133^{+}} \geq 80 \%$ & $88.9 \% \pm 4.04 \%$ & $88.1 \% \pm 2.3 \%$ \\
\hline
\end{tabular}


Table 4

DP holding time after thawing

\begin{tabular}{|c|c|c|c|c|c|c|c|c|}
\hline $\begin{array}{l}\text { ATMP } \\
\text { (manufacturer) }\end{array}$ & $\begin{array}{l}\text { Thawing } \\
\text { method }\end{array}$ & Post-thaw processing & $\begin{array}{l}\text { Shelf life } \\
\text { assigned upon } \\
\text { thawing ( } \mathrm{n}^{\circ} \\
\text { batches tested) }\end{array}$ & $\begin{array}{l}\text { QC test } \\
\text { performed to } \\
\text { assign shelf life }\end{array}$ & Test method & $\begin{array}{l}\text { Test } \\
\text { specifications }\end{array}$ & $\begin{array}{l}\text { QC test result } \\
\text { before freezing }\end{array}$ & $\begin{array}{l}\text { QC test result at } \\
\text { latest time point } \\
\text { tested after thawing }\end{array}$ \\
\hline BM-MSC (CF2) & $37^{\circ} \mathrm{C}$ water bath & $\begin{array}{l}\text { Administered } \\
\text { immediately }\end{array}$ & $15 \min (n=3)$ & Viability & Trypan Blue & $\geq 70 \%$ viability & $95.8 \% \pm 1.1 \%$ & $91.1 \% \pm 4.5 \%$ \\
\hline BM-MSC (CF5) & $37^{\circ} \mathrm{C}$ water bath & $\begin{array}{l}\text { Infused after dilution in } \\
\mathrm{NaCl} 0.9 \%+4 \% \mathrm{HSA}\end{array}$ & $4 \mathrm{~h}(\mathrm{n}=3)$ & Viability & Trypan Blue & $\geq 80 \%$ viability & $\begin{array}{c}100 \% \pm 0.0 \% \\
(96.0 \% \pm \\
7.0 \%)^{*}\end{array}$ & $85.0 \% \pm 9.0 \%$ \\
\hline CB-MSC (CF3) & $\begin{array}{l}37^{\circ} \mathrm{C} \text { water bath } \\
\text { or dry thaw- } \\
\text { ing device }\end{array}$ & $\begin{array}{l}\text { Dilution } 1: 1 \text { in } \mathrm{NaCl} \\
\quad 0.9 \%+10 \% \mathrm{HSA}+12 \% \\
\text { ACD-A }\end{array}$ & $30 \min (n=3)$ & $\begin{array}{l}\text { Viability } \\
\text { Cytofluorimetry }\end{array}$ & Cytofluorimetry & \multirow[t]{3}{*}{$\geq 80 \%$ viability } & \multirow[t]{3}{*}{$95.3 \% \pm 0.6 \%$} & $\begin{array}{l}82.3 \% \pm 1.5 \% \\
\text { Immunophenotype } \\
\text { CD } 45^{-} \text {CD } 90^{+} \text {CD } 105^{+} \\
\quad \geq 90 \%\end{array}$ \\
\hline \multirow[t]{2}{*}{$\begin{array}{l}\text { Proliferation } \\
\text { Potential }\end{array}$} & $\begin{array}{l}\text { Gentian Violet } \\
\text { staining }\end{array}$ & colony formation & $16 \% \pm 6.6 \%$ & $\begin{array}{l}94.8 \% \pm 3.6 \% \\
16 \% \pm 6.1 \%\end{array}$ & $94.5 \% \pm 3.0 \%$ & & & \\
\hline & Cell count & Fold Increase $>2$ & $22.0 \% \pm 12.1 \%$ & $25.0 \% \pm 19.3 \%$ & & & & \\
\hline CTL (CF5) & $37^{\circ} \mathrm{C}$ water bath & $\begin{array}{l}\text { Infused after dilution in } \\
\mathrm{NaCl} 0.9 \%+4 \% \mathrm{HSA}\end{array}$ & $1 \mathrm{~h}(\mathrm{n}=3)$ & Viability & Trypan Blue & $\geq 70 \%$ viability & $\begin{array}{l}94.0 \% \pm 1.7 \% \\
(83.0 \% \pm \\
5.0 \%)^{*}\end{array}$ & $81.0 \% \pm 7.0 \%$ \\
\hline LTC-ADV (CF5) & $37^{\circ} \mathrm{C}$ water bath & $\begin{array}{l}\text { Infused after dilution in } \\
\mathrm{NaCl} 0.9 \%+4 \% \mathrm{HSA}\end{array}$ & $2 \mathrm{~h}(\mathrm{n}=3)$ & Viability & Trypan Blue & $\geq 70 \%$ viability & $\begin{array}{l}100 \% \pm 0.0 \% \\
(91.0 \% \pm \\
1.7 \%)^{*}\end{array}$ & $79.0 \% \pm 2.5 \%$ \\
\hline $\begin{array}{l}\text { DENDR-1 } \\
\text { DENDR-2 } \\
(\mathrm{CF} 4)\end{array}$ & $37^{\circ} \mathrm{C}$ water bath & $\begin{array}{l}\text { Administered } \\
\text { immediately }\end{array}$ & $20 \min (n=3)$ & Viability & Trypan Blue & $\geq 75 \%$ viability & $98.2 \% \pm 0.2 \%$ & $96.3 \% \pm 1.0 \%$ \\
\hline
\end{tabular}

*At thawing.

cryopreserved DPs and a very stringent $\geq 90 \%$ in 1 case. These specifications were demonstrated to be maintained for all ATMPs at the longest storage time point tested (Table 2). Results of the time course of viability assays, performed on several ATMPs representative of different cell types, are shown in Figure 1. In the case of CIK (Figure 1D), the data derive from analyses from two different cell factories (CF1 and CF2). In
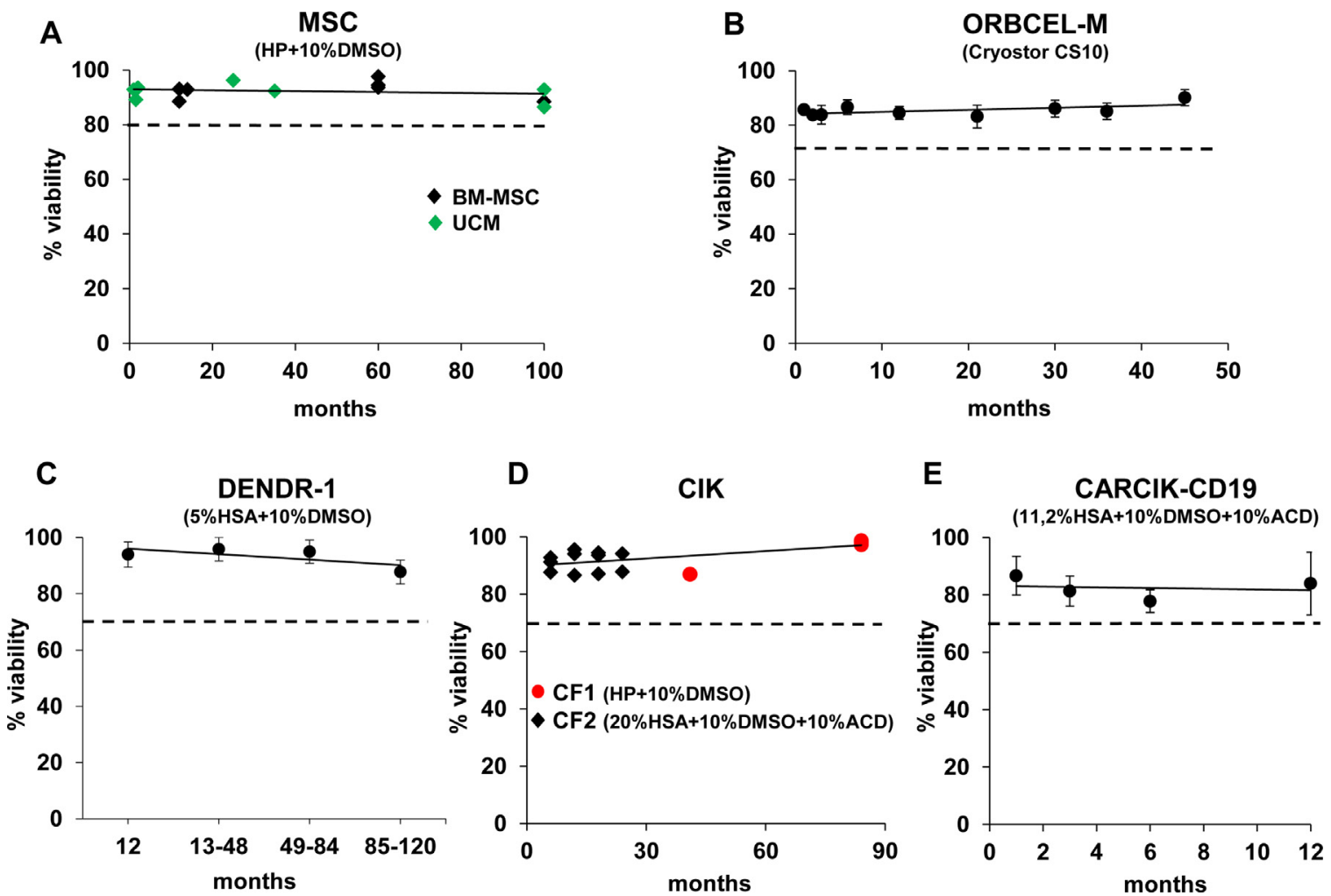

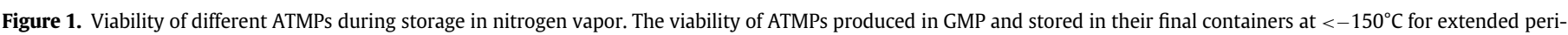

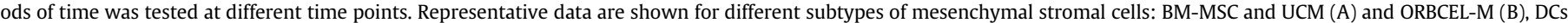

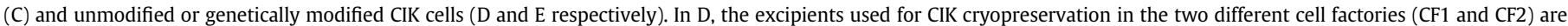

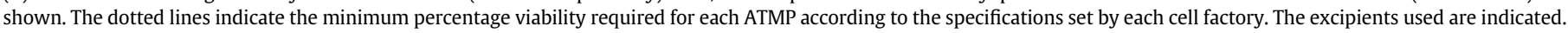
At least three batches of each ATMP were used in the viability studies, all time points included. (Color version of figure is available online.) 


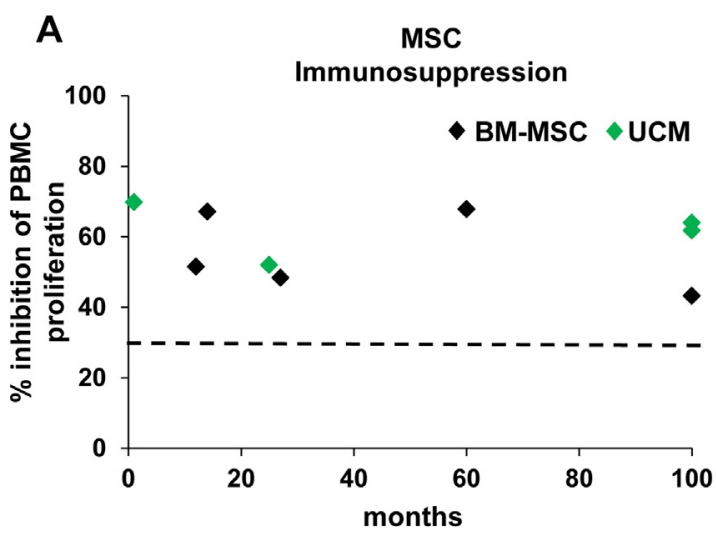

C

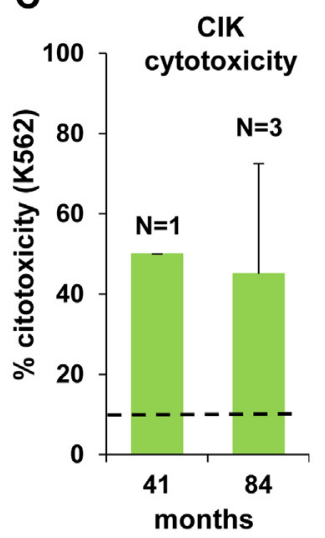

D

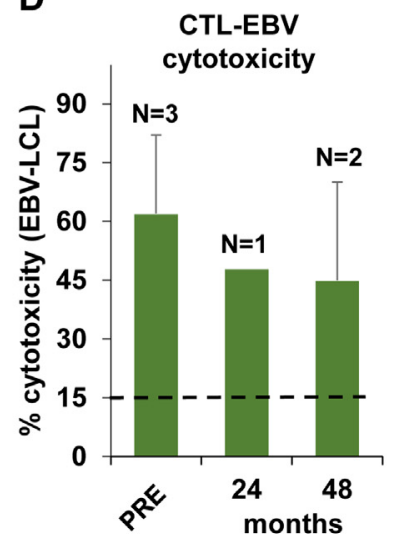

B

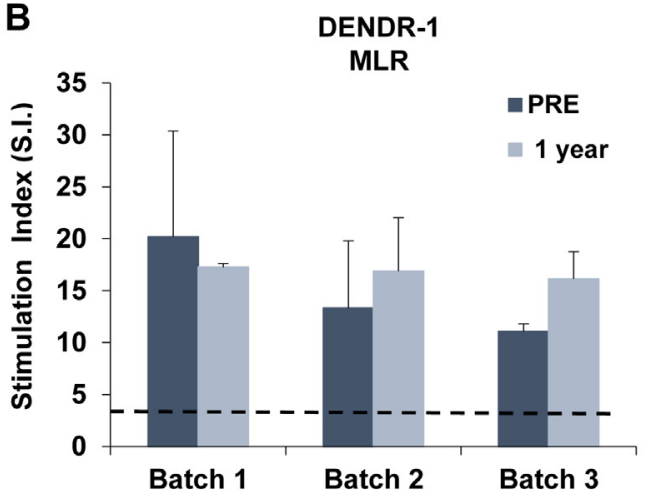

E

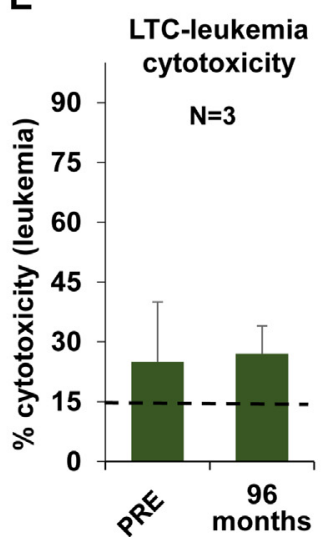

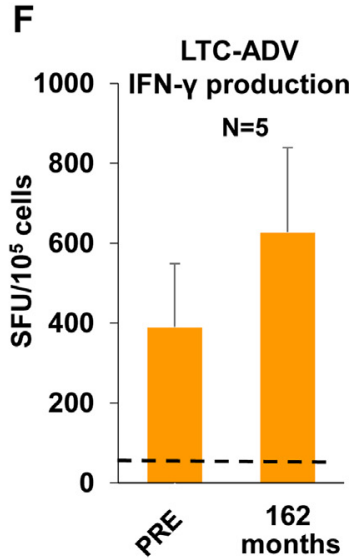

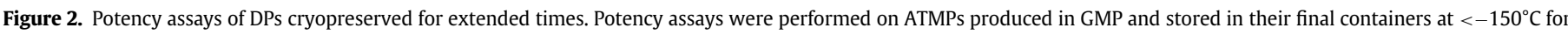

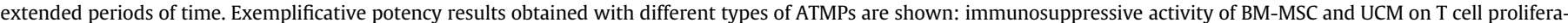

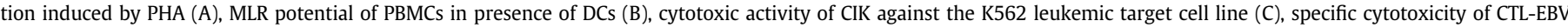

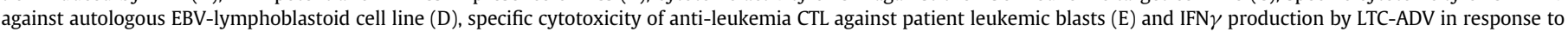
adenovirus peptides (F). At least three batches of each ATMP were used overall in the potency studies. (Color version of figure is available online.)

this case, however, the same testing method was used (cytofluorimetry), and the DP was produced according to the same procedure and used in a multicenter clinical trial involving both cell factories (NCT01186809, Table 1). Of note is that the trend lines that fit the viability data are almost flat and show a very limited downward trend. In addition, they remain always well above the thresholds even for extended periods, suggesting that the viability of the DPs should be maintained above the established limit for at least 10 to 13 years, and this was observed for all products tested in long-term studies (Figure 1 and Table 2).

Like viability, in all cases tested, ATMPs stored for extended periods at $<-150^{\circ} \mathrm{C}$ maintained their initial identity and purity (immunophenotype), without any loss or tendency for diminished expression of specific markers over time, even after the longest tested period of up to 13.5 years (Supplementary Figure S1 and Table 2).

Potency assays were performed in vitro for 13 out of 19 cryopreserved ATMPs. These included the capacity to mediate immunosuppression, $\mathrm{T}$ cell activation, cytotoxicity, cytokine production in presence of leukemic or cell targets expressing viral antigens, proliferation and differentiation potential (Table 2 and Material and Methods). The results show that, as for viability, identity and purity, there was no significant loss of potency, nor any tendency for a significant reduction over time, even over extended periods of up to 13.5 years (Figure 2 and Table 2). Aspects of ATMP safety were not always included in stability studies, owing to lack of clear guidelines. For three of 11 MSCbased ATMPs, karyotype analysis was performed after cryopreservation [27]. These results were found to be according to specifications.

More frequently, microbiologic contamination during storage was investigated, an unlikely but possible event that may occur in case of defective containers or their deterioration over time [29]. Thus, for 14 of
19 cryopreserved ATMPs, bacterial sterility was tested and, for seven, also mycoplasma and endotoxin contamination, the latter a marker of some kinds of contamination. In all cases analyzed, the maintenance of sterility could be validated for freezing bags or cryovials for 1 to 13,5 years (Table 2 ).

\section{Stability studies of fresh DPs}

Some ATMPs cannot be cryopreserved at the end of production. Stability of five of these DPs was analyzed, with the aim to establish whether the cells were still viable after a storage at $2^{\circ}$ to $8^{\circ} \mathrm{C}$, for the length of time necessary before infusion into patients, considering worst-case situations. The data obtained show that stability of noncryopreserved DPs, washed and resuspended in infusion medium, can be assigned a 4- to 24-h shelf life, based on results of viability (five of five), immunophenotyping (four of five) and in one case potency assay (proliferation potential in vitro and cell differentiation in vivo) (Table 3). In two cases, sterility after 24-h storage was also verified, since containers were in this case syringes and therefore potentially less protected from the environment.

\section{Stability studies on cryopreserved intermediates}

Past guidelines have suggested the need to control the stability of cryopreserved intermediates that are generated during expansion of some ATMPs, such as MSCs, and stored in cryovials. We collected the stability studies performed in the five cell factories on cell intermediates, and the details of the most complete data are available as Supplementary Table S1. The tests performed include in all cases (three 
of three) viability, immunophenotype and bacterial sterility. Cell recovery (one of three) and proliferation capacity (two of three) were also investigated. Shelf lives of 1 to 9 years could be assigned to different cryopreserved intermediates, confirming the long-term stability of cryopreserved intermediates.

\section{Holding time of DPs after thawing}

The cryopreserved cells need to be thawed before infusion into patients. Although the procedures plan for immediate infusion, stability testing has been introduced to define a maximal holding time for the thawed DP that takes into account the need to perform quality controls before infusion or transport to the clinical unit. Viability of the cells is again the most frequently tested attribute verified in stability studies of thawed DPs, but in some cases immunophenotype and capacity to expand in culture or form colonies has also been investigated (Table 4). The data generated on five DPs allow us to define a maximal holding time upon thawing ranging from 15 min to $4 \mathrm{~h}$, specific for each ATMP in its excipient storage temperature and container (Table 4).

\section{Discussion}

In this report, we collected the results of stability studies performed on 19 different experimental ATMPs, belonging to at least five cell lineages and produced over the last 13 years in GMP conditions in five authorized academic cell factories in the context of phase I/II clinical trials [30-48]. The stability studies were performed mostly on the final cryopreserved DPs in their primary containers.

The data presented show that all cryopreserved experimental ATMPs maintained, when stored at $<-150^{\circ} \mathrm{C}$ in different freezing media containing $10 \%$ DMSO, a very high level of viability over very long periods of time. Viability, cell identity and purity were maintained above the specifications for $\leq 13.5$ years, and the trend did not indicate any significant decrease in viability and identity over these longest tested periods. The results of functional potency assays, carried out on $68 \%$ of stored DPs, confirmed the viability data [49]. The data presented are not surprising, since many other cell products or tissues are known to maintain high viability and functionality for extended times when resuspended in iso-osmotic solutions containing $10 \%$ DMSO and cryopreserved in nitrogen vapor [50-53]. Successful long-term storage of cell-based medicines for therapeutic transplantation has been extensively demonstrated over many years, for example, hematopoietic stem cells used in the context of hematopoietic transplantation, which can be conserved in a cryopreserved state for $\leq 20$ years $[54,55]$. Human embryos stored at $<-150^{\circ} \mathrm{C}$ for $\leq 12$ years have been shown to be fully viable and implant successfully in utero, giving rise to healthy babies $[56,57]$. Worth noting is that the storage temperature was monitored continuously, and DPs were maintained continuously in the same nitrogen tanks throughout their storage period and were likely subject only to limited temperature oscillations, except during their manual insertion and retrieval $[58,59]$. Clearly, it is important that remote monitoring and alarm systems should be in place for the continuous assessment of tank functionality in cell factories, and comprehensive quality management procedures on cryostorage of ATMPs should also be established and routinely followed to mitigate the possible negative consequences of out-of-specification events [60].

The data here are important because they have been performed on multiple ATMPs produced by five cell factories over an extended period of time, and similar studies on this type of DPs have not been published so far. The results suggest that stability studies for novel ATMPs may not need to have extensive analyses at short time points $(<1 \mathrm{y})$, but rather should verify and validate longer periods of storage. This is important in the planning of these studies, in which many aliquots of several batches of ATMPs need to be generated and set aside for long-term storage and analysis. Although it is true that many ATMPs are dedicated to single patients (in particular autologous or HLA-matched ATMPs) and generally infused in patients within $1 \mathrm{y}$ of production, others can be given regardless of donor's HLA and are off-the-shelf products, for which validated long-term stability may be a useful characteristic. Stability studies are quite demanding, requiring multiple aliquots of at least three batches of ATMPs and the execution of a number of biological tests, including complex potency assays.

The International Council for Harmonization of Technical Requirements for Pharmaceuticals for Human Use (ICH) has established stability testing guidelines that provide comprehensive guidance on registration stability requirements for new drugs in the ICH regions (Europe, United States and Japan) [6,61]. These guidelines suggest stability testing of DPs every 3 months during the first year of storage, every 6 months over the second year and once a year thereafter. The testing plan also has to consider possible variables such as different drug concentrations and the need to test at least three batches. Although a matrixing design can be implemented to reduce the numbers of aliquots and tests to be performed [62], these studies are in any case very time consuming and costly. They also need to be repeated every time an excipient or container is modified or any other significant change is introduced to the cryopreservation procedure. However, in the absence of specific guidelines for cryopreserved ATMPs, practices vary between laboratories, as also evidenced in this report, and regulatory authorities performing inspections often require a time plan that is in line with the rules set for standard drugs. Reduced testing of drugs during the first years of ATMP storage, as suggested by the data presented, as well as harmonized practices would therefore facilitate cell factories and the development of ATMPs [4].

Another aspect of stability testing is the need to guarantee the sterility of the ATMPs throughout their shelf life, and therefore their safety [63,64]. Microbiologic controls should be part of stability study plans, in consideration of the fact that containers may lose their seal during time $[29,65,66]$. We show here that different DPs cryopreserved in different excipients in freezing bags with overwrap bags, or in cryovials, always maintained sterility for up to 13,5 years. This result is not particularly surprising, at least for freezing bags, which have a double barrier to contamination, which should avoid problems due to possible bag failures $[29,67]$. Sterility of products may indeed depend on the type and quality of the primary containers and perhaps to some extent on the excipients or additive present, but are unlikely to depend on the specific ATMPs. We therefore suggest that a novel risk-based matrixing approach could be used for sterility testing in the context of stability studies: a cell factory may be able to validate specific excipients and containers in long-term studies, by including in their analyses all the different tests available, including sterility, mycoplasma and endotoxin. We propose that, once specific excipients, additives and container have been validated within a cell factory for absence of microbial contamination in long-term studies, such tests would not be required to be repeated extensively for each new ATMP. Such a risk-based approach would provide a high level of guarantee and would reduce costs by avoiding the necessity of repeating all the tests on each ATMP, unless significant changes in containers, excipients, additives or storage methods are introduced.

Finally, an important aspect emerging from the present report is that there is a need to harmonize the quality control test methods, in particular potency assays, as well as define acceptable specifications, in particular for viability parameters.

To conclude, we believe that the results presented here are the most extensive analysis of stability studies of ATMPs published so far. On the basis of the scientific evidence presented, we propose that specific guidelines regarding stability studies and shelf life assignments for this type of DP should be introduced, that should (1) better define the type of tests that need to be performed for ATMPs (at a 
minimum viability and immunophenotype, but also potency studies, at least at some time points); (2) revise significantly the frequency of stability testing for ATMPs-we would suggest that testing of viability, identity and potency at an early time point (e.g., 1 month after freezing) should be sufficient to control the effect of the freezing procedure on these parameters and to assign a 1-year shelf life, if the 1month testing is within specifications; stability testing for the same parameters could then be repeated at 1 year and every 2 years thereafter, and shelf life could be extended at each test by 2 years in presence of results within the expected specifications; (3) test the microbiological attributes on stored DPs after a risk analysis, and thus tests need not be repeated on all ATMPs or for all attributes, provided the same containers and excipients/additives and freezing procedures are used and appropriate times have already been tested at least in triplicate; (4) investigate holding times with viability studies for non-cryopreserved DPs and upon thawing of cryopreserved products, with possible matrixing for DPs belonging to the same cell types and conserved in the same solutions. All these changes would need to be justified within the stability plan after a risk-based analysis. A longer shelf life assigned outright to all cryopreserved ATMPs is consistent with other practices, for example, shelf life assigned to cryopreserved heart valves [50].

We strongly believe that such specific guidelines would harmonize practices between different laboratories and guarantee full quality of the ATMPs during the assigned shelf lives but also simplify stability studies and significantly reduce costs. Similar studies should be analyzed for other cell-based intermediates used by the industry, in particular for hybridomas or recombinant eukaryotic cells used for therapeutic antibodies and cytokine production, because simplified procedures may be put in place to reduce costs without diminishing safety.

\section{Acknowledgments}

This work has been fully supported by Fondazione Regionale per la Ricerca Biomedica (FRRB, Regione Lombardia), Project $\mathrm{N}^{\circ} \mathrm{CP} 2$ _10/ 2018 (Plagencell).

The GMP productions of cells used for the stability studies analyzed here were however part of several clinical trials funded by: European Union's Horizon 2020 research and innovation program under grant agreements $\mathrm{N}^{\circ} 634086$ (NEPHSTROM), H2020-SC1 20162017 and ORTHOUNION Project (under G.A. 733288), European Union's 7th Framework Programme (FP7 HEALTH-2009-1.4-2) with the REBORNE Project (under G.A. 241876); the Independent Research 2016 AIFA with the RACE study protocol (Code.AIFA-2016-02364896) and Haplo-CIK (code AIFA-2016-02364730); Amgen Ltd, Cambridge, UK (for the BET 2017 study). The materials presented and views expressed here are the responsibility of the authors(s) only. The EU Commission takes no responsibility for any use made of the information set out. .

We thank Elisa Gotti, Carolina Cuofano, Olga Pedrini, Rut Valgardsdottir, Irene Cattaneo, Silvia Zaninelli, (Center of Cellular Therapy “G. Lanzani”, ASST Papa Giovanni XXIII Bergamo), Giada Matera, Stefania Cesana, Valentina Colombo (Laboratory of Cellular and Gene Therapy S. Verri, ASST Monza), Simona Pogliani (Cell Therapy Production Unit, IRCCS Istituto Neurologico Carlo Besta, Milano), Rosaria Giordano, Tiziana Montemurro, Cristiana Lavazza, Elisa Montelatici (Laboratorio di Medicina Rigenerativa, Fondazione IRCCS Ca' Granda Ospedale Maggiore Policlinico, Milano), Dr. Antonia Moretta, Daniela Montagna, Maria Antonietta Avanzini e Sabrina Basso (UOSD Cell Factory, Fondazione IRCCS Policlinico San Matteo, Pavia) for their participation to the stability studies described in this report. We finally thank Andrew Finnerty (Centre for Cell Manufacturing Ireland, National University of Ireland, Galway, Ireland), Jon Smythe (NHS Blood and Transplant Advanced Therapies Unit, Liverpool, UK) and Helene Roelofs (Leiden University Medical Centre, Leiden, The
Netherlands) for their participation to the production and stability studies of the ATMP ORBCEL-M described in part in this report.

\section{Supplementary materials}

Supplementary material associated with this article can be found in the online version at doi:10.1016/j.jcyt.2021.12.004.

\section{References}

[1] Jiskoot W. Advanced Therapy Medicinal Products: What's in a Name? Journal of pharmaceutical sciences 2020;109(11):3282-4

[2] Regulation (EC) No 1394/ 2007, in: E.p.a.t. Council (Ed.) https://eur-lex.europa.eu/ legal-content/IT/TXT/PDF/?uri=CELEX:32007R1394\&from=EN.

[3] Hubel A, Spindler R, Skubitz AP. Storage of human biospecimens: selection of the optimal storage temperature. Biopreservation and biobanking 2014;12(3): $165-75$.

[4] Iyer RK, Bowles PA, Kim H, Dulgar-Tulloch A. Industrializing Autologous Adoptive Immunotherapies: Manufacturing Advances and Challenges. Frontiers in medicine 2018;5:150

[5] A. Hubel, Preservation of Cells: A Practical Manual, Wiley Blackwell 2018.

[6] International Council on Harmonisation. Stability testing for new drug substances and products. ICH Q1A(R2), 2003, https://database.ich.org/sites/default/files/ Q1A\%28R2\%29\%20Guideline.pdf.

[7] Galli MC. ATMPs for Cancer Immunotherapy: A Regulatory Overview. Methods in molecular biology 2016;1393:1-9

[8] European Directive 2001 /83/CE, in: E. Commission (Ed.) https://ec.europa.eu/ health.

[9] Directive 2009 /120/EC, https://ec.europa.eu/health/sites/default/files/files eudralex/vol-1/dir_2009_120/dir_2009_120_en.pdf.

[10] Guidelines on good manufacturing Practice specific to advanced therapy medicinal products 2017, https://www.ema.europa.eu/en/news/new-guidelines-goodmanufacturing-practices-advanced-therapies.

[11] FDA Guidance for Industry: Stability Testing of Drug Substances and Drug Products. 1998, https://www.fda.gov/media/71707/download.

[12] Aashigari S, Ramya Goud R, Sneha S, Vykuntam U, Potnuri NR. Stability studies of pharmaceutical products. World Journal of Pharmaceutical Research 2018;8 (1):479-92.

[13] Haider K, Akash MSH, Faheem A, Rehman K. Guidelines for drug stability and stability testing. In: Akash MSH, Rehman K, eds. Drug Stability and Chemical Kinetics, Eds., Springer; 2020:19-29 https://www.springer.com/gp/book/ 9789811564253.

[14] Food and Drug Administration, 21 CFR 312.23(a)(7)(ii), https://www.govinfo.gov/ app/details/CFR-2011-title21-vol5/CFR-2011-title21-vol5-sec312-23.

[15] Rajan R, Matsumura K. Development and Application of Cryoprotectants, 1081 Advances in experimental medicine and biology; 2018. p. 339-54.

[16] Clarke DM, Yadock DJ, Nicoud IB, Mathew AJ, Heimfeld S. Improved post-thaw recovery of peripheral blood stem/progenitor cells using a novel intracellular-like cryopreservation solution. Cytotherapy 2009;11(4):472-9.

[17] Ginis I, Grinblat B, Shirvan MH. Evaluation of bone marrow-derived mesenchyma stem cells after cryopreservation and hypothermic storage in clinically safe medium. Tissue engineering. Part C, Methods 2012;18(6):453-63.

[18] Stylianou J, Vowels M. Hadfield K. Novel cryoprotectant significantly improves the post-thaw recovery and quality of HSC from CB. Cytotherapy 2006;8(1): 57-61.

[19] Vigano M, Budelli S, Lavazza C, Montemurro T, Montelatici E, de Cesare S, Lazzari L, Orlandi AR, Lunghi G, Giordano R. Tips and Tricks for Validation of Quality Control Analytical Methods in Good Manufacturing Practice Mesenchymal Stromal Cell Production. Stem cells international 2018;2018:3038565.

[20] Lisini D, Nava S, Frigerio S, Pogliani S, Maronati G, Marcianti A, Cocce V, Bondiolotti G, Cavicchini L, Paino F, Petrella F, Alessandri G, Parati EA, Pessina A. Automated Large-Scale Production of Paclitaxel Loaded Mesenchymal Stromal Cells for Cell Therapy Applications. Pharmaceutics 2020;12(5).

[21] Capelli C, Zaccara E, Cipriani P, Di Benedetto P, Maglione W, Andracco R, Di Luca C, Pignataro F, Giacomelli R, Introna M, Vitali C, Del Papa N. Phenotypical and Functional Characteristics of In Vitro-Expanded Adipose-Derived Mesenchymal Stromal Cells From Patients With Systematic Sclerosis. Cell transplantation 2017;26 (5):841-54.

[22] Comoli P, Schilham MW, Basso S, van Vreeswijk T, Bernardo ME, Maccario R, van Tol MJ, Locatelli F, Veltrop-Duits LA. T-cell lines specific for peptides of adenovirus hexon protein and devoid of alloreactivity against recipient cells can be obtained from HLA-haploidentical donors. Journal of immunotherapy 2008;31(6):529-36.

[23] Brennan MA, Renaud A, Amiaud J, Rojewski MT, Schrezenmeier H, Heymann D, Trichet V, Layrolle P. Pre-clinical studies of bone regeneration with human bone marrow stromal cells and biphasic calcium phosphate. Stem cell research \& therapy 2014;5(5):114.

[24] Barilani M, Lavazza C, Vigano M, Montemurro T, Boldrin V, Parazzi V, Montelatici E, Crosti M, Moro M, Giordano R, Lazzari L. Dissection of the cord blood stroma component reveals predictive parameters for culture outcome. Stem cells and development 2015;24(1):104-14.

[25] Nava S, Lisini D, Pogliani S, Dossena M, Bersano A, Pellegatta S, Parati E, Finocchiaro G, Frigerio S. Safe and Reproducible Preparation of Functional Dendritic 
Cells for Immunotherapy in Glioblastoma Patients. Stem cells translational medicine 2015;4(10):1164-72.

[26] Veronesi E, Murgia A, Caselli A, Grisendi G, Piccinno MS, Rasini V, Giordano R, Montemurro T, Bourin P, Sensebe L, Rojewski MT, Schrezenmeier H, Layrolle P, Ginebra MP, Panaitescu CB, Gomez-Barrena E, Catani F, Paolucci P, Burns JS, Dominici M. Transportation conditions for prompt use of ex vivo expanded and freshly harvested clinical-grade bone marrow mesenchymal stromal/stem cells for bone regeneration. Tissue engineering. Part C, Methods 2014;20(3):239-51.

27] Capelli C, Pedrini O, Cassina G, Spinelli O, Salmoiraghi S, Golay J, Rambaldi A, Giussani U, Introna M. Frequent occurrence of non-malignant genetic alterations in clinical grade mesenchymal stromal cells expanded for cell therapy protocols. Haematologica 2014;99(6):e94-7.

[28] Gaipa G, Introna M, Golay J, Nolli ML, Vallanti G, Parati E, Giordano R, Romagnoli L, Melazzini M, Biondi A, Biagi E. Development of advanced therapies in Italy: Management models and sustainability in six Italian cell factories. Cytotherapy 2016;18(4):481-6

[29] Khuu HM, Cowley H, David-Ocampo V, Carter CS, Kasten-Sportes C, Wayne AS, Solomon SR, Bishop MR, Childs RM, Read EJ. Catastrophic failures of freezing bags for cellular therapy products: description, cause, and consequences. Cytotherapy 2002:4(6):539-49.

[30] Casiraghi F, Perico N, Podesta MA, Todeschini M, Zambelli M, Colledan M, Camagni S, Fagiuoli S, Pinna AD, Cescon M, Bertuzzo V, Maroni L, Introna M Capelli C, Golay JT, Buzzi M, Mister M, Ordonez PYR, Breno M, Mele C, Villa A, Remuzzi G, Group M-LS. Third-party bone marrow-derived mesenchymal stromal cell infusion before liver transplantation: A randomized controlled trial. American journal of transplantation : official journal of the American Society of Transplantation and the American Society of Transplant Surgeons 2021;21(8):2795-809.

[31] Uccelli A, Laroni A, Brundin L, Clanet M, Fernandez O, Nabavi SM, Muraro PA, Oliveri RS, Radue EW, Sellner J, Soelberg Sorensen P, Sormani MP, Wuerfel JT, Battaglia MA, Freedman MS. M.s. group, MEsenchymal StEm cells for Multiple Sclerosis (MESEMS): a randomized, double blind, cross-over phase I/II clinical trial with autologous mesenchymal stem cells for the therapy of multiple sclerosis. Trials 2019;20(1):263.

[32] Introna M, Lussana F, Algarotti A, Gotti E, Valgardsdottir R, Mico C, Grassi A, Pavoni C, Ferrari ML, Delaini F, Todisco E, Cavattoni I, Deola S, Biagi E, Balduzzi A, Rovelli A, Parma M, Napolitano S, Sgroi G, Marrocco E, Perseghin P, Belotti D, Cab iati B, Gaipa G, Golay J, Biondi A, Rambaldi A. Phase II Study of Sequential Infusion of Donor Lymphocyte Infusion and Cytokine-Induced Killer Cells for Patients Relapsed after Allogeneic Hematopoietic Stem Cell Transplantation. Biology of blood and marrow transplantation : journal of the American Society for Blood and Marrow Transplantation 2017;23(12):2070-8.

[33] Introna M, Lucchini G, Dander E, Galimberti S, Rovelli A, Balduzzi A, Longoni D, Pavan F, Masciocchi F, Algarotti A, Mico C, Grassi A, Deola S, Cavattoni I, Gaipa G, Belotti D, Perseghin P, Parma M, Pogliani E, Golay J, Pedrini O, Capelli C, Cortelazzo S. D'Amico G, Biondi A, Rambaldi A, Biagi E. Treatment of graft versus host disease with mesenchymal stromal cells: a phase I study on 40 adult and pediatric patients. Biology of blood and marrow transplantation : journal of the American Society for Blood and Marrow Transplantation 2014;20(3):375-81.

[34] Bassetti B, Carbucicchio C, Catto V, Gambini E, Rurali E, Bestetti A, Gaipa G, Belotti D, Celeste F, Parma M, Righetti S, Biava L, Arosio M, Bonomi A, Agostoni P, Scacciatella P, Achilli F, Pompilio G. Linking cell function with perfusion: insights from the transcatheter delivery of bone marrow-derived CD133(+) cells in ischemic refractory cardiomyopathy trial (RECARDIO). Stem cell research \& therapy 2018;9(1):235.

[35] Comoli P, Pedrazzoli P, Maccario R, Basso S, Carminati O, Labirio M, Schiavo R, Secondino S, Frasson C, Perotti C, Moroni M, Locatelli F, Siena S. Cell therapy of stage IV nasopharyngeal carcinoma with autologous Epstein-Barr virus-targeted cytotoxic T lymphocytes. Journal of clinical oncology : official journal of the American Society of Clinical Oncology 2005;23(35):8942-9.

[36] Eoli M, Corbetta C, Anghileri E, Di Ianni N, Milani M, Cuccarini V, Musio S, Paterra R, Frigerio S, Nava S, Lisini D, Pessina S, Maddaloni L, Lombardi R, Tardini M, Ferroli P, DiMeco F, Bruzzone MG, Antozzi C, Pollo B, Finocchiaro G, Pellegatta S. Expansion of effector and memory $\mathrm{T}$ cells is associated with increased survival in recurrent glioblastomas treated with dendritic cell immunotherapy. Neurooncology advances 2019;1(1). vdz022.

[37] Perico N, Casiraghi F, Todeschini M, Cortinovis M, Gotti E, Portalupi V, Mister M Gaspari F, Villa A, Fiori S, Introna M, Longhi E, Remuzzi G. Long-Term Clinical and Immunological Profile of Kidney Transplant Patients Given Mesenchymal Stromal Cell Immunotherapy. Frontiers in immunology 2018;9:1359.

[38] Magnani CF Gaipa G, Lussana F, Belotti D Gritti G, Napolitano S, Matera G, Cabiat B, Buracchi C, Borleri G, Fazio G, Zaninelli S, Tettamanti S, Cesana S, Colombo V, Quaroni M, Cazzaniga G, Rovelli A, Biagi E, Galimberti S, Calabria A, Benedicenti F, Montini E, Ferrari S, Introna M, Balduzzi A, Valsecchi MG, Dastoli G, Rambaldi A Biondi A. Sleeping Beauty-engineered CAR T cells achieve antileukemic activity without severe toxicities. The Journal of clinical investigation 2020;130 (11):6021-33

[39] Comoli P, Basso S, Zecca M, Pagliara D, Baldanti F, Bernardo ME, Barberi W, Moretta A, Labirio M, Paulli M, Furione M, Maccario R, Locatelli F. Preemptive therapy of EBV-related lymphoproliferative disease after pediatric haploidentical stem cell transplantation. American journal of transplantation : official journal of the American Society of Transplantation and the American Society of Transplant Surgeons 2007;7(6):1648-55.

[40] Perico N, Casiraghi F, Introna M, Gotti E, Todeschini M, Cavinato RA, Capelli C, Rambaldi A, Cassis P, Rizzo P, Cortinovis M, Marasa M, Golay J, Noris M, Remuzzi G. Autologous mesenchymal stromal cells and kidney transplantation: a pilot study of safety and clinical feasibility. Clinical journal of the American Society of Nephrology : CJASN 2011;6(2):412-22.
[41] Perico N, Casiraghi F, Gotti E, Introna M, Todeschini M, Cavinato RA, Capelli C, Rambaldi A, Cassis P, Rizzo P, Cortinovis M, Noris M, Remuzzi G. Mesenchymal stromal cells and kidney transplantation: pretransplant infusion protects from graft dysfunction while fostering immunoregulation, Transplant international : official journal of the European Society for. Organ Transplantation 2013;26(9):867-78.

[42] Introna $M$, Borleri $G$, Conti $E$, Franceschetti M, Barbui AM, Broady R, Dander E, Gaipa G, D'Amico G, Biagi E, Parma M, Pogliani EM, Spinelli O, Baronciani D, Grassi A, Golay J, Barbui T, Biondi A, Rambaldi A. Repeated infusions of donor-derived cytokine-induced killer cells in patients relapsing after allogeneic stem cell transplantation: a phase I study. Haematologica 2007:92(7):952-9.

[43] Belingheri M, Lazzari L, Parazzi V, Groppali E, Biagi E, Gaipa G, Giordano R, Rastaldi MP, Croci D, Biondi A, Rebulla P, Edefonti A, Ghio L. Allogeneic mesenchymal stem cell infusion for the stabilization of focal segmental glomerulosclerosis. Biologicals : journal of the International Association of Biological Standardization 2013;41(6):439-45

[44] Gomez-Barrena E, Padilla-Eguiluz NG, Rosset P, Hernigou P, Baldini N, Ciapetti G, Gonzalo-Daganzo RM, Avendano-Sola $C$, Rouard $H$, Giordano R, Dominici M Schrezenmeier H, Layrolle P, On Behalf Of The Reborne C. Osteonecrosis of the Femoral Head Safely Healed with Autologous, Expanded, Bone Marrow-Derived Mesenchymal Stromal Cells in a Multicentric Trial with Minimum 5 Years FollowUp. Journal of clinical medicine 2021;10(3)

[45] Gomez-Barrena E, Padilla-Eguiluz N, Rosset P, Gebhard F, Hernigou P, Baldini N, Rouard H, Sensebe L, Gonzalo-Daganzo RM, Giordano R, Garcia-Rey E, CorderoAmpuero J, Rubio-Suarez IC, Garcia-Simon MD, Stanovici J, Ehrnthaller C, HuberLang M, Flouzat-Lachaniette CH, Chevallier N, Donati DM, Spazzoli B, Ciapetti G, Fleury S, Fernandez MN, Cabrera JR, Avendano-Sola C, Montemurro T, Panaitescu C, Veronesi E, Rojewski MT, Lotfi R, Dominici M, Schrezenmeier H, Layrolle P. Early efficacy evaluation of mesenchymal stromal cells (MSC) combined to biomaterials to treat long bone non-unions. Injury 2020;51:S63-73. Suppl 1.

[46] Ball LM, Bernardo ME, Roelofs H, van Tol MJ, Contoli B, Zwaginga JJ, Avanzini MA Conforti A, Bertaina A, Giorgiani G, Jol-van der Zijde CM, Zecca M, Blanc KLe, Frassoni F, Egeler RM, Fibbe WE, Lankester AC, Locatelli F. Multiple infusions of mesenchymal stromal cells induce sustained remission in children with steroidrefractory, grade III-IV acute graft-versus-host disease. British journal of haematology 2013;163(4):501-9.

[47] Ciccocioppo R, Bernardo ME, Sgarella A, Maccario R, Avanzini MA, Ubezio C, MineIli A, Alvisi C, Vanoli A, Calliada F, Dionigi P, Perotti C, Locatelli F, Corazza GR. Autologous bone marrow-derived mesenchymal stromal cells in the treatment of fistulising Crohn's disease. Gut 2011;60(6):788-98

[48] Comoli P, Basso S, Riva G, Barozzi P, Guido I, Gurrado A, Quartuccio G, Rubert L, Lagreca I, Vallerini D, Forghieri F, Morselli M, Bresciani P, Cuoghi A, Paolini A, Colaci E, Marasca R, Cuneo A, Iughetti L, Trenti T, Narni F, Foa R, Zecca M, Luppi M, Potenza L. BCR-ABL-specific T-cell therapy in Ph+ ALL patients on tyrosine-kinase inhibitors. Blood 2017;129(5):582-6.

[49] International Conference on Harmonisation of Technical Requirements for Registration of Pharmaceuticals for Human Use. Specifications: Test Procedures and Acceptance Criteria for Biotechnological/Biological Products Q6B, 1999, https:// database.ich.org/sites/default/files/Q6B\%20Guideline.pdf.

[50] Burkert J, Kochova P, Tonar Z, Cimrman R, Blassova T, Jashari R, Fiala R, Spatenka J. The time has come to extend the expiration limit of cryopreserved allograft heart valves. Cell and tissue banking 2020.

[51] Underwood J, Rahim M, West C, Britton R, Skipworth E, Graves V, Sexton S, Harris H, Schwering D, Sinn A, Pollok KE, Robertson KA, Goebel WS, Hege KM. How old is too old? In vivo engraftment of human peripheral blood stem cells cryopreserved for up to 18 years - implications for clinical transplantation and stability programs. World journal of stem cells 2020;12(5):359-67.

[52] Mareschi K, Adamini A, Castiglia S, Rustichelli D, Castello L, Mandese A, Leone M, Pinnetta G, Mesiano G, Ferrero I, Fagioli F. Cytokine-Induced Killer (CIK) Cells, In Vitro Expanded under Good Manufacturing Process (GMP) Conditions, Remain Stable over Time after Cryopreservation. Pharmaceuticals 2020;13(5).

[53] Shafa M, Walsh T, Panchalingam KM, Richardson T, Menendez L, Tian X, Suresh Babu S, Dadgar S, Beller J, Yang F, Baghbaderani BA. Long-Term Stability and Differentiation Potential of Cryopreserved cGMP-Compliant Human Induced Pluripotent Stem Cells. International journal of molecular sciences 2019;21(1)

[54] Lisenko K, Pavel P, Kriegsmann M, Bruckner T, Hillengass J, Goldschmidt H, Witzens-Harig M, Ho AD, Wuchter P. Storage Duration of Autologous Stem Cell Preparations Has No Impact on Hematopoietic Recovery after Transplantation. Biology of blood and marrow transplantation : journal of the American Society for Blood and Marrow Transplantation 2017;23(4):684-90.

[55] Kim GH, Kwak J, Kim SH, Kim HJ, Hong HK, Jin HJ, Choi SJ, Oh W, Um S. High Integrity and Fidelity of Long-Term Cryopreserved Umbilical Cord Blood for Transplantation. Journal of clinical medicine 2021;10(2).

[56] Revel A, Safran A, Laufer N, Lewin A, Reubinov BE, Simon A. Twin delivery following 12 years of human embryo cryopreservation: case report. Human reproduction 2004;19(2):328-9,

[57] Yuan Y, Mai Q Ma J, Deng M, Xu Y, Zhuang G, Zhou C. What was the fate of human embryos following long-term cryopreservation ( $>1=12$ years) and frozen embryo transfer? Human reproduction 2019;34(1):52-5

[58] Angel S, von Briesen H, Oh YJ, Baller MK, Zimmermann H, Germann A. Toward Optimal Cryopreservation and Storage for Achievement of High Cell Recovery and Maintenance of Cell Viability and T Cell Functionality. Biopreservation and biobanking 2016;14(6):539-47.

[59] Pogozhykh D, Pogozhykh O, Prokopyuk V, Kuleshova L, Goltsev A, Blasczyk R, Mueller T. Influence of temperature fluctuations during cryopreservation on vital parameters, differentiation potential, and transgene expression of placental multipotent stromal cells. Stem cell research \& therapy 2017;8(1):66. 
[60] Schiewe MC, Freeman M, Whitney JB, VerMilyea MD, Jones A, Aguirre M, Leisinger C, Adaniya G, Synder N, Chilton R, Behnke EJ. Comprehensive assessment of cryogenic storage risk and quality management concerns: best practice guidelines for ART labs. Journal of assisted reproduction and genetics 2019;36(1):5-14.

[61] International Council on Harmonisation. Stability testing of Biotechnological/Biological Products. ICH Q5C, 1995, https://database.ich.org/sites/default/files/ Q5C\%20Guideline.pdf.

[62] International Council on Harmonisation. Matrixing and Bracketing designs for stability Testing of New Drug Substances and Product. ICH Q1D, 2002, https:// database.ich.org/sites/default/files/Q1D\%20Guideline.pdf.

[63] Brown H, Mahler HC, Mellman J, Nieto A, Wagner D, Schaar M, Mathaes R, Kossinna J, Schmitting F, Dreher S, Roehl H, Hemminger M, Wuchner K. Container Closure Integrity Testing-Practical Aspects and Approaches in the Pharmaceutical Industry. PDA journal of pharmaceutical science and technology 2017;71(2):147-62.
[64] Golay J, Pedrini O, Capelli C, Gotti E, Borleri G, Magri M, Vailati F, Passera M, Farina C, Rambaldi A, Introna M. Utility of routine evaluation of sterility of cellular therapy products with or without extensive manipulation: Best practices and clinical significance. Cytotherapy 2018;20(2):262-70.

[65] Nieto A, Roehl H, Brown H, Nikoloff J, Adler M, Mahler HC. Evaluation of Container Closure System Integrity for Frozen Storage Drug Products. PDA journal of pharmaceutical science and technology 2016;70(2):120-33.

[66] Jere D, Sediq AS, Huwyler J, Vollrath I, Kardorff M, Mahler HC. Challenges for CellBased Medicinal Products From a Pharmaceutical Product Perspective. Journal of pharmaceutical sciences 2021;110(5):1900-8.

[67] Becherucci V, Bisin S, Ermini S, Piccini L, Gori V, Gentile F, Ceccantini R, De Rienzo E, Bindi B, Pavan P, Cunial V, Allegro E, Brugnolo F, Bambi F. Comparison of CryoMACS Freezing Bags with Maco Biotech Freezing-Ethinyl Vinyl Acetate Bags for Hematopoietic Progenitor Cells Cryopreservation Using a CD34(+)-Enriched Product. Biopreservation and biobanking 2020;18(5):454-61. 